MPP-2007-34

\title{
Perturbative and nonperturbative correspondences between compact and non-compact sigma-models
}

\author{
M. Niedermaier*, E. Seiler, P. Weisz \\ Laboratoire de Mathématiques et Physique Théorique \\ CNRS/UMR 6083, Université de Tours \\ Parc de Grandmont, 37200 Tours, France \\ Max-Planck-Institut für Physik \\ Föhringer Ring 6 \\ 80805 München, Germany
}

\begin{abstract}
Compact (ferro- and antiferromagnetic) sigma-models and noncompact (hyperbolic) sigma-models are compared in a lattice formulation in dimensions $d \geq 2$. While the ferro- and antiferromagnetic models are essentially equivalent, the qualitative difference to the noncompact models is highlighted. The perturbative and the large $N$ expansions are studied in both types of models and are argued to be asymptotic expansions on a finite lattice. An exact correspondence between the expansion coefficients of the compact and the noncompact models is established, for both expansions, valid to all orders on a finite lattice. The perturbative one involves flipping the sign of the coupling and remains valid in the termwise infinite volume limit. The large $N$ correspondence concerns the functional dependence on the free propagator and holds directly only in finite volume.
\end{abstract}

${ }^{*}$ Membre du CNRS 


\section{Introduction}

Nonlinear sigma-models with maximally symmetric riemannian target spaces naturally come in dual pairs, one compact and the other noncompact. The generalized classical spin systems associated with both the compact and the noncompact target spaces have a variety of applications, see e.g. [1, 2, 3] for the less familiar noncompact models. Since the compact models are much better understood and the target manifolds can be related to those of the noncompact models by analytic continuation, it is natural to try to relate also the quantum (or statistical mechanics) properties of the compact to that of the noncompact models. For definiteness we consider here as target-spaces the $N$-dimensional sphere $\mathbb{S}^{N}$ and its dual the hyperboloid $\mathbb{H}^{N}$, however the qualitative aspects should be the same for other dual pairs.

The goal of this note is to present two examples of such relations for invariant correlation functions. The first one concerns perturbation theory, the other the large $N$ expansion. Provided one has chosen a formulation where the expansions are valid asymptotic expansions, it turns out that knowledge of the expansion coefficients in the compact model allows one to infer those in the noncompact model. However it is crucial that the asymptotic expansions are known to exist beforehand in both systems independently. The lattice formulation is especially suited to address this and we shall see that on a finite lattice the relevant asymptotic expansions do exist; for the perturbative one in section 2 below and for the large $N$ expansion in a separate paper [4]. The perturbative correspondence simply involves a sign flip of the coefficients and in a formal expansion (dimensional regularization and minimal subtraction) has been noted to low orders in $[5,6]$ and in the literature on Riemannian sigma models. Here we present a proof of the correspondence to all loop orders, initially on a finite lattice; but the termwise infinite volume limit should exist on account of the expected lattice counterpart of David's theorem $[7,8]$. The perturbatively defined correlation functions, viewed as functions of the lattice points and of $1 / \beta$, are thus likewise related simply by flipping the sign of $\beta$.

The large $N$ correspondence is more subtle. In brief the $s$-th order large $N$ coefficient $W_{ \pm, r}^{(s)}$ of the invariant $2 r$-point functions on a finite lattice can in the compact $(+)$ and in the noncompact (-) model be expressed in terms of a single functional $X_{r}^{(s)}[D](\lambda)$ of the leading order invariant two-point function $D$ - the same functional for both systems. This "large $N$ correspondence" is computationally useful because the computations in the compact model, which do not require gauge fixing, are much simpler. The 'detour' over finite volume cannot be avoided as the correspondence is difficult to interpret directly in the infinite volume limit, see [9]. In addition is important to appreciate that although the gap equations are related by flipping the sign of the large $N$ coupling $\lambda=(N+1) / \beta$, the leading order propagator $D_{-}$in the noncompact model behaves very differently as a function of the lattice distance than $D_{+}$: while the latter shows exponential decay in the thermodynamic limit, the former decreases only with a power law (for $d>2$ ) or increases logarithmically with the distance (for $d=2$ ).

In a finite volume the gap equations for the compact as well as the noncompact model 
have the same $O(V)$ number of solutions for the (mass) ${ }^{2}$ parameter; but whereas in the compact model there is exactly one positive solution, which is the 'physical' one, in addition to multiple negative ones, the sign-flipped gap equation has only negative solutions of which only one is the right one for defining $D_{-}$. The fundamental criterion by which the relevant solution is selected should be the stability of the corresponding saddle point, but we find also an alternative characterization selecting the physically relevant saddle point of the noncompact model. In the thermodynamic limit, in both cases all the negative solutions of the gap equation disappear, therefore the large $N$ expanded correlation functions, viewed as functions of the lattice points, are not related in any simple way, in particular not merely by flipping the sign of $\lambda$.

The derivations of the above results also highlight why one cannot expect useful correspondences to exist beyond asymptotic expansions. For example flipping the sign of the coupling in the (exact) generating functionals for invariant correlation functions maps the ferromagnet onto the compact antiferromagnet. In contrast, in the perturbative asymptotic expansion the sign flip rather relates the ferromagnet to the noncompact model, while in the large $N$ expansion the functional dependence on the independently defined free propagators gets related. In the latter case the relation to the compact model cannot be formulated directly in the thermodynamic limit but one can take term by term the thermodynamic limit on both sides of the correspondence. This somewhat tricky limit is studied in an accompanying paper [9] in the two-dimensional systems for a number of physically interesting invariant quantities. Ultimately we expect the fundamental differences between the compact and the noncompact models to be rooted in two facts: the presence of a infinite volume mass gap and the absence of long range order (for $d \leq 2$ ) in the compact models and the opposite characteristics in the noncompact models for all $d \geq 1$.

The rest of the paper is organized as follows: In section 2 we introduce generating functionals for the invariant correlators in the three systems considered and establish the perturbative correspondence. In section 3 dual formulations of the generating functions are introduced, suited for the large $N$ expansions. The large $N$ correspondence is shown first from the generating functionals, then from the Schwinger-Dyson equations, and finally verified at low orders.

\section{Invariant correlators and PT correspondence}

Invariant correlators in the noncompact model have to be defined in terms of a gauge fixed generating functional. A gauge fixing where one spin is kept fixed turns out to be advantageous. To discuss the relation to the compact model we adopt the same gauge fixing there. This spin model formulation is also convenient to discuss the relation between the exact generating functionals and for the proof of the perturbative (PT) correspondence. 


\subsection{Definitions}

We begin by setting up the notation and the definitions for the invariant correlation functions considered and their generating functionals. We consider the $\mathrm{O}(N+1)$ spherical and the $\mathrm{SO}(1, N)$ hyperbolic sigma-models with standard lattice action, defined on a hypercubic lattice $\Lambda \subset \mathbb{Z}^{d}$ of volume $V=|\Lambda|=L^{d}$. The dynamical variables ("spins") will be denoted by $n_{x}^{a}, x \in \Lambda, a=0, \ldots, N$, in both cases, and periodic boundary conditions are assumed throughout $n_{x+L \hat{\mu}}=n_{x}$. The constraint is $n \cdot n=1$ in both cases, but with different 'dot' products; namely $a \cdot b:=a^{0} b^{0}+a^{1} b^{1}+\ldots+a^{N} b^{N}=: a^{c} \delta_{c d} b^{d}$ in the compact model, and $a \cdot b:=a^{0} b^{0}-a^{1} b^{1}-\ldots-a^{N} b^{N}=: a^{c} \eta_{c d} b^{d}$ in the noncompact model. We shall also use the notation $\vec{a}=\left(a^{1}, \ldots, a^{N}\right)$ for vectors in $\mathbb{R}^{N}$, so that the bilinear forms read $a \cdot b=a^{0} b^{0} \pm \vec{a} \cdot \vec{b}$, in the two cases. Clearly $\mathbb{S}^{N}=\left\{n \in \mathbb{R}^{N+1} \mid n \cdot n=1\right\}$ is the $N$-sphere and $\mathbb{H}^{N}=\left\{n \in \mathbb{R}^{1, N} \mid n \cdot n=1, n^{0}>0\right\}$ is the upper half of the twosheeted $N$-dimensional hyperboloid. The invariance groups are $\mathrm{O}(N+1)$ and $\mathrm{SO}_{0}(1, N)$, respectively.

Let us briefly note how $\mathbb{S}^{N}$ and $\mathbb{H}^{N}$ are related by symmetric space duality (see for instance $[10,11])$. Recall that a symmetric space $G / K$ has an involution $\tau$ associated with it such that the Lie algebra $\mathfrak{g}$ of $G$ decomposes according to $\mathfrak{g}=\mathfrak{k} \oplus \mathfrak{m}$ as a direct sum of vector spaces, where $\mathfrak{k}$ and $\mathfrak{m}$ are even and odd under $\tau$, respectively. Furthermore $[\mathfrak{k}, \mathfrak{k}] \subset \mathfrak{k},[\mathfrak{k}, \mathfrak{m}] \subset \mathfrak{m}$ and $[\mathfrak{m}, \mathfrak{m}] \subset \mathfrak{k}$. The dual Lie algebra $\mathfrak{g}^{*}$ is then defined as $\mathfrak{g}^{*}:=\mathfrak{k} \oplus i \mathfrak{m}$ and the corresponding dual group $G^{*}$ is the (simply connected) group whose Lie algebra is $\mathfrak{g}^{*} . G^{*}$ contains a connected subgroup $\widetilde{K}$ having $\mathfrak{k}$ as its Lie algebra; the dual symmetric space $(G / K)^{*}$ can then be defined as $G^{*} / \widetilde{K}$. On $\mathfrak{g}$ we have an invariant bilinear form $B$ (in our case simply minus the Killing form) which induces a dual bilinear form $B^{*}$ on $\mathfrak{g}^{*}$. The bilinear forms $B$ and $-B^{*}$, restricted to $\mathfrak{m}$ and $i \mathfrak{m}$, respectively, are positive definite in our case and define the metric of the tangent spaces at the origins of the symmetric spaces $G / K$ and $(G / K)^{*}$, respectively. By requiring invariance under $G$ or $G^{*}$, respectively, Riemannian metrics on both symmetric spaces are induced. For $G=\mathrm{SO}(N+1), K=\mathrm{SO}(N)$, this gives $G^{*}=\widetilde{\mathrm{SO}}_{0}(1, N)$ (the universal covering group of $\left.\mathrm{SO}_{0}(1, N)\right)$ and $(G / K)^{*}=\widetilde{\mathrm{SO}_{0}}(1, N) / \widetilde{\mathrm{SO}_{0}}(N)=\mathrm{SO}_{0}(1, N) / \mathrm{SO}_{0}(N)$ or $G / K=\mathbb{S}^{N}$ and $(G / K)^{*}=\mathbb{H}^{N}$.

The lattice actions for the two systems are

$$
S_{ \pm}=\mp \beta \sum_{x, \mu}\left(n_{x} \cdot n_{x+\hat{\mu}}-1\right)=\mp \frac{\beta}{2} \sum_{x} n_{x} \cdot(\Delta n)_{x}
$$

where the upper sign refers to the compact model and the lower sign to the noncompact model. In the compact model, with the conventions adopted in (2.3), $\beta>0$ models ferromagnetic behavior while $\beta<0$ models antiferromagnetic behavior. In the noncompact model only $\beta>0$ is allowed and the action is unbounded from above, $0 \leq S_{-}[n]<\infty$. 
The Laplacian is $\Delta_{x y}=-\sum_{\mu}\left[2 \delta_{x, y}-\delta_{x, y+\hat{\mu}}-\delta_{x, y-\hat{\mu}}\right]$, as usual. We write

$$
\begin{aligned}
& d \Omega_{+}(n)=d n^{N+1} \delta(n \cdot n-1), \\
& d \Omega_{-}(n)=2 d n^{N+1} \delta(n \cdot n-1) \theta\left(n^{0}\right),
\end{aligned}
$$

for the invariant measure on $\mathbb{S}^{N}$ and $\mathbb{H}^{N}$, respectively. Further $\delta_{ \pm}\left(n, n^{\prime}\right)$ is the invariant point measure on $\mathbb{S}^{N}, \mathbb{H}^{N}$, and $n^{\uparrow}=(1,0, \ldots, 0)$. Note that the measure $d \Omega_{+}(n)$ is normalized while $\mathbb{H}^{N}$ has infinite volume.

In the compact model we consider two generating functionals, the usual one and a variant with one spin frozen:

$$
\begin{aligned}
\exp W[H] & =\mathcal{N} \int \prod_{x} d \Omega_{+}\left(n_{x}\right) \exp \left\{-S_{+}+\frac{1}{2} \sum_{x, y} H_{x y}\left(n_{x} \cdot n_{y}-1\right)\right\} \\
\exp W_{+}[H] & =\mathcal{N}_{+} \int \prod_{x} d \Omega_{+}\left(n_{x}\right) \delta_{+}\left(n_{x_{0}}, n^{\uparrow}\right) \exp \left\{-S_{+}+\frac{1}{2} \sum_{x, y} H_{x y}\left(n_{x} \cdot n_{y}-1\right)\right\}
\end{aligned}
$$

where $H_{x y} \geq 0$ is a source field and the normalizations $\mathcal{N}, \mathcal{N}_{+}$are such that $W[0]=$ 0 . More generally $W_{n}[H]$ will denote the generating functional with the spin at site $x_{0}$ fixed by a $\delta\left(n_{x_{0}}, n\right)$ insertion. In this notation one has $W_{+}[H]=W_{n^{\uparrow}}[H]$ and $\int d \Omega_{+}(n) \exp W_{n}[H]=\exp W[H]$. In fact

$$
W_{n}[H]=W[H]
$$

for all $n \in \mathbb{S}^{N}$. This can seen by performing a global rotation $n_{x} \mapsto g n_{x}=: \tilde{n}_{x}, x \in \Lambda$, with $g \in \mathrm{O}(N+1)$ chosen such that $g n_{x_{0}}=n^{\uparrow}$, say. The Boltzmann factor will then depend on $\tilde{n}_{x}, x \neq x_{0}$, only and the $d \Omega\left(\tilde{n}_{x_{0}}\right)=d \Omega\left(n_{x_{0}}\right)$ integration can be performed to give 1.

In the noncompact model only the fixed spin variant of the generating functional is well defined and we write

$$
\exp W_{-}[H]=\mathcal{N}_{-} \int \prod_{x} d \Omega_{-}\left(n_{x}\right) \delta_{-}\left(n_{x_{0}}, n^{\uparrow}\right) \exp \left\{-S_{-}+\frac{1}{2} \sum_{x, y} H_{x y}\left(n_{x} \cdot n_{y}-1\right)\right\}
$$

where now $H_{x y}<0$ sources give damping exponentials.

Partially connected $2 r$ point functions are defined by

$$
\begin{aligned}
& W_{ \pm}[H]=\sum_{r \geq 1} \frac{1}{r ! 2^{r}} W_{ \pm, r}\left(x_{1}, y_{1} ; \ldots ; x_{r}, y_{r}\right) H_{x_{1} y_{1}} \ldots H_{x_{r} y_{r}} \\
& W_{ \pm, r}\left(x_{1}, y_{1} ; \ldots ; x_{r}, y_{r}\right):=\left.h_{x_{1} y_{1}} \ldots h_{x_{r} y_{r}} W_{ \pm}[H]\right|_{H=0}, \quad h_{x y}:=\frac{\delta}{\delta H_{x y}} .
\end{aligned}
$$


In particular $W_{ \pm, 1}(x, y)=\left\langle n_{x} \cdot n_{y}\right\rangle-1, W_{ \pm, 2}\left(x_{1}, y_{1} ; x_{2}, y_{2}\right):=\left\langle n_{x_{1}} \cdot n_{y_{1}} n_{x_{2}} \cdot n_{y_{2}}\right\rangle-\left\langle n_{x_{1}}\right.$. $\left.n_{y_{1}}\right\rangle\left\langle n_{x_{2}} \cdot n_{y_{2}}\right\rangle$, where \langle\rangle are the functional averages with respect to $\mathcal{N}_{ \pm}^{-1} e^{-S_{ \pm}}$. Note that $W_{ \pm, r}(\ldots ; x, x ; \ldots)=0$.

In the above we tacitly assumed that $W_{ \pm}[H]$ and the correlation functions computed from it do not depend on the site $x_{0}$ of the frozen spin and are translation invariant. We show now that this indeed the case. If we momentarily indicate the dependence on the site as $W_{x_{0}}$ (and drop the \pm subscripts) one has trivially

$$
W_{x_{0}}\left[\tau_{a} H\right]=W_{x_{0}+a}[H], \quad\left(\tau_{a} H\right)_{x y}=H_{x+a, y+a} .
$$

Thus, if $W_{x_{0}}$ is independent of $x_{0}$ it is also translation invariant. The Boltzmann factors $(2.3 \mathrm{~b})$ and $(2.5)$ can be viewed as a function on the group via $F\left(g_{0}, \ldots, g_{s}\right)=$ $f\left(g_{0} n^{\uparrow}, \ldots, g_{s} n^{\uparrow}\right)$, where we picked some ordering of the sites $x_{i}, i=0,1, \ldots, s:=V-1$, identified $n_{x_{i}}$ with $g_{i} n^{\uparrow}$, and wrote momentarily $f$ for the Boltzmann factor. Then $W_{x_{i}}$ is of the form

$$
\begin{gathered}
\int \prod_{j} d \Omega\left(n_{j}\right) \delta\left(n_{i}, n^{\uparrow}\right) f\left(n_{0}, \ldots, n_{s}\right)=\text { const } U_{i}, \\
U_{i}:=\int \prod_{j \neq i} d g_{j} F\left(g_{0}, \ldots, g_{i-1}, e, g_{i+1}, \ldots, g_{s}\right) .
\end{gathered}
$$

Using the invariance of $F$ under $g_{i} \mapsto h^{-1} g_{i}$ and the unimodularity and invariance of the measure $d g$ one verifies that $U_{i}=U_{0}$ for all $i$.

A peculiarity of the fixed spin gauge is that an invariant $2 r$-spin correlator $\left\langle n_{x_{1}} \cdot n_{y_{1}} n_{x_{2}}\right.$. $\left.n_{y_{2}} \ldots n_{x_{r}} \cdot n_{y_{r}}\right\rangle$, can be re-interpreted as a non-invariant $2 r-1$-spin correlator. This is manifest when $x_{i}=x_{0}$ for some $i$; since $n_{x_{0}}=n^{\uparrow}$ the correlator then involves only $2 r-1$ fluctuating spins. By the above argument any one site can play the role of $x_{0}$, so that picking such a re-interpretation amounts to picking a site where $n_{x_{i}}=n^{\uparrow}$. This feature holds both in the compact and in the noncompact models, but initially only on a finite lattice. When the site $x_{0}$ is kept fixed in the interior of the lattice, these specific noninvariant $2 r-1$ point functions will have a pointwise thermodynamic limit in both cases. However the fixed spin averages then do not approach limits which can be interpreted in terms of averages without gauge fixing or with a translation invariant gauge fixing. This changes if $x_{0}$ is identified with a point on the boundary and moves out to infinity as $\Lambda \rightarrow \mathbb{Z}^{d}$. In this case, in dimensions $d \leq 2$ an important difference between the compact and noncompact models emerges: while in the compact model the MerminWagner theorem assures that the non-invariant correlator is equal to its invariant average over $\mathrm{O}(N+1)$, in the noncompact model the non-amenability of $\mathrm{SO}(1, N)$ prevents this, and we find either spontaneous symmetry breaking or divergence of the correlators, as discussed in [1].

The goal in the following will be to relate the perturbative and the large $N$ expansions in the compact and the noncompact models. For the perturbative expansions of the 
correlation functions, we write

$$
W_{ \pm, r} \sim \beta^{-r} \sum_{n \geq 0} \beta^{-n} w_{ \pm, r}^{(n)}
$$

In a large $N$ expansion $\lambda:=(N+1) / \beta$ is kept fixed and the coefficient functions $W_{ \pm, r}^{(s)}$ in

$$
W_{ \pm, r} \sim \frac{\lambda^{r}}{(N+1)^{r-1}} \sum_{s \geq 0} \frac{1}{(N+1)^{s}} W_{ \pm, r}^{(s)}
$$

are sought.

\subsection{Compact antiferromagnet and noncompact model}

In the literature one finds statements, based on formal manipulations of functional integrals, suggesting that compact and noncompact models are essentially related just by flipping the sign of $\beta$ (in [2], however, the qualifying remark is added that such a relation is 'less clear' at the non-perturbative level). Since this simple 'flip rule' is not completely correct and the relations between the compact model, its analytic continuation to $\beta<0$ and the noncompact model are somewhat subtle, let us try to clarify the situation. To avoid confusion, in the following $\beta$ will always be assumed to be non-negative.

The compact model defined in Eq. (2.1) describes for $\beta>0$ a ferromagnet, for $\beta<0$ an antiferromagnet, so flipping the sign of $\beta$ turns a ferromagnet into an antiferromagnet, not the noncompact model. It turns out that one can nevertheless relate the coefficients of the perturbation expansion and the large $N$ expansion of the compact ferromagnetic and the noncompact model by a sign flip prescription, which does, however, not imply any simple relation between the antiferromagnet and the noncompact model.

On the simple hypercubic lattices we are considering here, there is a transformation between the cases $\beta>0$ and $\beta<0$, based on the fact that we can decompose $\Lambda$ into an even and an odd sub-lattice

$$
\Lambda=\Lambda_{+} \cup \Lambda_{-},
$$

where $x \in \Lambda_{+}$if and only if $\sum_{i=1}^{d} x_{i}$ is even, otherwise $x \in \Lambda_{-}$. Defining

$$
\epsilon_{x}:=(-1)^{x_{1}+\ldots+x_{d}}
$$

we can define a map of the configurations by

$$
n_{x} \mapsto\left(n_{x}^{\prime}\right)=\epsilon_{x} n_{x}
$$


mapping each configuration of the ferromagnet into one of the antiferromagnet with the same (correctly normalized) Boltzmann weight. This yields the following identity relating the generating functionals of the antiferromagnetic and the ferromagnetic systems:

$$
W_{+,-\beta}[H]=W_{+, \beta}\left[H^{\epsilon}\right]+\frac{1}{2} \sum_{x y}\left(H_{x y}^{\epsilon}-H_{x y}\right)+\mathrm{const},
$$

where we momentarily indicated the dependence on $\beta$ and set $H_{x y}^{\epsilon}:=\epsilon_{x} H_{x y} \epsilon_{y}$. For the invariant two-point function this gives

$$
\left.\left\langle n_{x} \cdot n_{y}\right\rangle\right|_{-\beta}=\left.\epsilon_{x} \epsilon_{y}\left\langle n_{x} \cdot n_{y}\right\rangle\right|_{\beta},
$$

and similarly for the higher correlation functions. This implies that the physics of the antiferromagnet is essentially the same as for the ferromagnet, in particular they have the same mass gap, phase structure etc..

The relation between the ferromagnetic $\mathrm{O}(N+1)$ and the noncompact $\mathrm{SO}(1, N)$ systems (the latter only exists in the ferromagnetic version) is, however, more subtle: starting from the $\mathrm{O}(N+1)$ model we re-parameterize the variables $n_{x} \in \mathbb{S}^{N}$ by introducing $\beta=: g^{-1}$ and $\vec{n}_{x}=: \sqrt{g} \vec{\pi}_{x}$, so that

$$
n_{x} \cdot n_{y}=n_{x}^{0} n_{y}^{0}+g \vec{\pi}_{x} \cdot \vec{\pi}_{y}=\sigma_{x} \sigma_{y} \sqrt{1-g \vec{\pi}_{x}^{2}} \sqrt{1-g \vec{\pi}_{y}^{2}}+g \vec{\pi}_{x} \cdot \vec{\pi}_{y}
$$

where the $\sigma_{x}$ are variables taking the values \pm 1 . To obtain $W_{+}[H]$ in this parameterization one has to $(i)$ integrate over all $\vec{\pi}_{x}$ subject to the constraint $\vec{\pi}_{x}^{2}<\beta$ and (ii) sum over all $\sigma_{x}= \pm 1$ except $\sigma_{x_{0}}$, which is 1 . Explicitly

$$
\begin{aligned}
& \exp W_{+}[H]=\widetilde{\mathcal{N}}_{+} \sum_{\left\{\sigma_{x}\right\}} \delta_{1, \sigma_{x_{0}}} \int \prod_{x} d \vec{\pi}_{x} \delta\left(\vec{\pi}_{x_{0}}\right) \theta\left(|\beta|-\vec{\pi}_{x}^{2}\right)\left(1-g \vec{\pi}_{x}^{2}\right)^{-1 / 2} \\
& \times \exp \left\{-\frac{1}{2 g} \sum_{x, \mu}\left[\left(\sigma_{x} \sqrt{1-g \vec{\pi}_{x}^{2}}-\sigma_{x+\hat{\mu}} \sqrt{1-g \vec{\pi}_{x+\hat{\mu}}^{2}}\right)^{2}+g\left(\vec{\pi}_{x}-\vec{\pi}_{x+\hat{\mu}}\right)^{2}\right]\right\} \\
& \times \exp \left\{\frac{1}{2} \sum_{x, y} H_{x y}\left(\sigma_{x} \sigma_{y} \sqrt{1-g \vec{\pi}_{x}^{2}} \sqrt{1-g \vec{\pi}_{y}^{2}}+g \vec{\pi}_{x} \cdot \vec{\pi}_{y}-1\right)\right\} .
\end{aligned}
$$

For $\beta \rightarrow+\infty$ (ferromagnet) the term in which all $\sigma_{x}$ are equal dominates (for $\beta \rightarrow-\infty$ (antiferromagnet) instead the terms with alternating $\sigma_{x}$ dominate).

On the other hand, we may re-parameterize the noncompact model in a similar way by introducing $\vec{n}_{x}=\sqrt{g} \vec{\pi}_{x}$, but this time

$$
n_{x} \cdot n_{y}=n_{x}^{0} n_{y}^{0}-g \vec{\pi}_{x} \cdot \vec{\pi}_{y}=\sqrt{1+g \vec{\pi}_{x}^{2}} \sqrt{1+g \vec{\pi}_{y}^{2}}-g \vec{\pi}_{x} \cdot \vec{\pi}_{y} .
$$


Inserting this into the expression for $W_{-}[H]$ given in $(2.3 \mathrm{~b})$, we now obtain

$$
\begin{aligned}
& \exp W_{-}[H]=\widetilde{\mathcal{N}}_{-} \int \prod_{x} d \vec{\pi}_{x} \delta\left(\vec{\pi}_{x_{0}}\right)\left(1+g \vec{\pi}_{x_{0}}\right)^{-1 / 2} \\
& \times \exp \left\{\frac{1}{2 g} \sum_{x, \mu}\left[\left(\sqrt{1+g \vec{\pi}_{x}^{2}}-\sqrt{1+g \vec{\pi}_{x+\hat{\mu}}}\right)^{2}-g\left(\vec{\pi}_{x}-\vec{\pi}_{x+\hat{\mu}}\right)^{2}\right]\right\} \\
& \times \exp \left\{\frac{1}{2} \sum_{x, y} H_{x y}\left(\sqrt{1+g \vec{\pi}_{x}^{2}} \sqrt{1+g \vec{\pi}_{y}^{2}}-g \vec{\pi}_{x} \cdot \vec{\pi}_{y}-1\right)\right\} .
\end{aligned}
$$

Comparing now Eqs (2.17) and (2.19), we see that the partition function of the noncompact model is obtained from the one of the compact one by $(i)$ flipping the sign of $\beta$, (ii) dropping all terms except the one with all $\sigma_{x}=1$ and (iii) omitting the $\theta$ functions restricting the domain of integration.

So it is clear that the analytic continuation of the compact system to negative $\beta$ (the antiferromagnet) is not equivalent to the noncompact $\mathrm{SO}(1, N)$ system, as one might infer from a cursory reading of the literature (for instance [2]). This non-equivalence will be shown even more manifestly below. Within the framework of the SchwingerDyson Equations used in section 3.5 a noteworthy consequence is that even the exact $\mathrm{SD}$ equations do not determine their solution uniquely.

In the following sections we will see, however, that nevertheless the perturbation expansions in a finite volume of the ferromagnetic compact and the noncompact model are related simply by flipping the sign of $\beta$ (but without the introduction of the sign factor $\epsilon_{x}$ as in the antiferromagnet); furthermore we will derive a relation between the $1 / N$ expansions of the two models.

\subsection{Perturbative correspondence}

We now consider the perturbative expansions (2.9) of the correlation functions. On a finite lattice it turns out the perturbative series in the compact ferromagnetic and in the noncompact models are asymptotic expansions which are simply related by a sign flip in the coupling, $\beta \mapsto-\beta$; the perturbation expansion of the noncompact model is, however, not equal to that of the antiferromagnet, as one might guess from this.

Concretely

$$
w_{-, r}^{(n)}=(-)^{r+n} w_{+, r}^{(n)}, \quad \forall r \geq 1, n \geq 0,
$$

for the coefficients (2.9). The sign flip rule was stated explicitly by Hikami [6] (in a formal continuum expansion based on dimensional regularization) but was presumably known to other authors in special cases and at low orders, e.g. [5]. It has also been observed in the perturbative beta functional of Riemannian sigma models (using dimensional regularization and minimal subtraction) and can readily be verified to all loop orders in 
this framework. But to the best of our knowledge no general proof is available in print, so we present a simple proof here.

We set $Z_{ \pm}[H]:=\exp W_{ \pm}[H]$ and define 


$$
\begin{aligned}
& Z_{+}^{\mathrm{PT}}[H](\beta):=\int \prod_{x} d \vec{\pi}_{x} \delta\left(\vec{\pi}_{x_{0}}\right) \theta\left(\beta-\vec{\pi}_{x}^{2}\right)\left(1-g \vec{\pi}_{x}^{2}\right)^{-1 / 2} \\
& \times \exp \left\{-\frac{\beta}{2} \sum_{x, \mu}\left[\left(\sqrt{1-g \vec{\pi}_{x}^{2}}-\sqrt{1-g \vec{\pi}_{x+\hat{\mu}}^{2}}\right)^{2}+g\left(\vec{\pi}_{x}-\vec{\pi}_{x+\hat{\mu}}\right)^{2}\right]\right\} \\
& \times \exp \left\{\frac{1}{2} \sum_{x, y} H_{x y}\left(\sqrt{1-g \vec{\pi}_{x}^{2}} \sqrt{1-g \vec{\pi}_{y}^{2}}+g \vec{\pi}_{x} \cdot \vec{\pi}_{y}-1\right)\right\},
\end{aligned}
$$

and

$$
\begin{aligned}
& Z_{-}^{\mathrm{PT}}[H](\beta):=\int \prod_{x} d \vec{\pi}_{x} \delta\left(\vec{\pi}_{x_{0}}\right) \theta\left(\beta-\vec{\pi}_{x}^{2}\right)\left(1+g \vec{\pi}_{x}^{2}\right)^{-1 / 2} \\
& \times \exp \left\{\frac{\beta}{2} \sum_{x, \mu}\left[\left(\sqrt{1+g \vec{\pi}_{x}^{2}}-\sqrt{1+g \vec{\pi}_{x+\hat{\mu}}^{2}}\right)^{2}-g\left(\vec{\pi}_{x}-\vec{\pi}_{x+\hat{\mu}}\right)^{2}\right]\right\} \\
& \times \exp \left\{\frac{1}{2} \sum_{x, y} H_{x y}\left(\sqrt{1+g \vec{\pi}_{x}^{2}} \sqrt{1+g \vec{\pi}_{y}^{2}}-g \vec{\pi}_{x} \cdot \vec{\pi}_{y}-1\right)\right\} .
\end{aligned}
$$

$Z_{+}^{\mathrm{PT}}[H]$ is, up to a multiplicative constant, just the term of $Z_{+}[H]$ (Eq.(2.17)) in which $\sigma_{x}=1$, for all $x$. $Z_{-}^{\mathrm{PT}}[H]$ differs from $Z_{-}[H]$ in Eq. (2.19) by the presence of the $\theta$ functions and a multiplicative constant. We now state the

\section{Result:}

$$
Z_{ \pm}[H]=c_{ \pm}(\beta) Z_{ \pm}^{\mathrm{PT}}[H](\beta)+O\left(\beta^{-\infty}\right),
$$

where $c_{ \pm}(\beta)$ are normalization constants independent of $H$. Further the $Z_{ \pm}^{\mathrm{PT}}[H](\beta)$ have asymptotic expansions of the form

$$
Z_{ \pm}^{\mathrm{PT}}[H](\beta) \sim \sum_{n=0}^{\infty} a_{n}[H]( \pm \beta)^{-n}
$$

with identical coefficients $a_{n}[H]$ in both cases.

The existence of the asymptotic expansions (2.24) is a standard result of Laplace's method in asymptotic analysis (see for instance $[12,13]$ ). The relation $(2.23)$ expresses the irrelevance of boundaries away from the maximum as well as of the terms in which the $\sigma_{x}$ are not all equal. It follows from two facts:

Fact 1: On a finite lattice in the compact ferromagnetic model all terms in $Z_{+}[H]$ except the one with $\sigma_{x}=1$ are $O\left(\beta^{-\infty}\right)$. 
Fact 2: On a finite lattice in the noncompact model for any $x_{0} \in \Lambda$ and $\beta \geq \beta_{0}>0$ the expectation value

$$
\begin{aligned}
\left\langle\theta\left(\vec{n}_{z}^{2}-1\right)\right\rangle:= & \frac{1}{Z_{-}[H]} \int \prod_{x} d \Omega_{-}\left(n_{x}\right) \delta\left(n_{x_{0}}, n^{\uparrow}\right) \theta\left(\vec{n}_{z}^{2}-1\right) \\
& \times \exp \left\{-S_{-}+\frac{1}{2} \sum_{x, y} H_{x y}\left(n_{x} \cdot n_{y}-1\right)\right\},
\end{aligned}
$$

is bounded by $a \exp (-b \beta)$ with $a, b>0$.

Corollary: There are constants $a_{ \pm}, b_{ \pm}>0$ such that for $\beta>\beta_{0}$

$$
\left|\frac{Z_{ \pm}^{\mathrm{PT}}[H](\beta) \beta^{-N \frac{V-1}{2}}}{Z_{ \pm}[H](\beta)}-1\right| \leq a_{ \pm} \exp \left(-b_{ \pm} \beta\right)
$$

Both facts have essentially the same origin: the fact that the 'energy' in the omitted contributions is exponentially small. We give a detailed proof only of Fact 2 and add some comments on the proof of Fact 1.

Proof of Fact 2: We do the integrations in the following way: first we integrate over all configurations having a fixed value $s$ of the action and afterwards over $s$. Thus we can write

$$
Z_{-}[H](\beta)=\int d s e^{-\beta s} \rho_{H}(s)
$$

with some non-negative density function $\rho_{H}$. If we then can show that for any configuration violating the field cutoff the action is larger than $s_{0}$ ('energy bound'), it will follow that

$$
Z_{-}[H](\beta)\left\langle\theta\left(\vec{n}_{x}^{2}-1\right)\right\rangle \leq \int d s e^{-\beta s} \rho_{H}(s) \theta\left(s-s_{0}\right) .
$$

The right hand side is easily seen to be $\leq \exp \left(-\frac{\beta}{2} s_{0}\right) \times Z_{-}[H](\beta / 2)$. By standard asymptotic analysis (see for instance $[12,13]$ ) it follows that $Z_{-}[H](\beta) / Z_{-}[H](\beta / 2)$ goes to a finite limit as $\beta \rightarrow \infty$, and therefore

$$
\frac{Z_{-}[H](\beta / 2)}{Z_{-}[H](\beta)} \leq a,
$$

for $\beta \geq \beta_{0}$. So Eq. (2.28) implies Fact 2 .

It remains to prove the 'energy bound'. Since we are using the fixed spin gauge, we have $n_{x_{0}}=n^{\uparrow}$. Then by assumption there is a site $x_{1}$ with $n_{x_{1}}^{0} \geq \sqrt{2}$. Choose a path $\mathrm{P}$ of length $|P| \leq V$ from $x_{0}$ to $x_{1}$ and denote the part of the action corresponding to the path $\mathrm{P}$ by $s_{P}$. We claim that $s_{P}$ is minimized by moving in equal steps along the geodesic from $n_{x_{0}}=n^{\uparrow}$ to $n_{x_{1}}$, which implies

$$
s \geq s_{P} \geq \frac{c}{|P|} \geq \frac{c}{V}
$$


with some constant $c$.

To see the lower bound on $s_{P}$ it is sufficient to consider a path of three points, since the minimization condition is local. Thus we only have to minimize $e(n):=n_{0} \cdot n+n \cdot n_{1}$ over $n$ and show that the minimum is assumed for $n$ being the midpoint of the geodesic in $\mathbb{H}^{N}$ from $n_{0}$ to $n_{1}$. Choosing coordinates such that $n_{0}=n^{\uparrow}$ and $n_{1}=\left(\operatorname{ch} \theta_{1}, \operatorname{sh} \theta_{1}, 0, \ldots 0\right)$ with $\theta_{1} \geq 0$ we find

$$
e(n)=n^{0}\left(1+\operatorname{ch} \theta_{1}\right)-n^{1} \operatorname{sh} \theta_{1} \geq n^{0}\left(1+\operatorname{ch} \theta_{1}\right)-\sqrt{\left(n^{0}\right)^{2}-1} \operatorname{sh} \theta_{1} .
$$

Putting $n^{0}=\operatorname{ch} \theta$ this becomes

$$
e(n) \geq \operatorname{ch} \theta+\operatorname{ch}\left(\theta_{1}-\theta\right) \geq 2 \operatorname{ch} \frac{\theta_{1}}{2}
$$

using the convexity of ch function in the last step. It follows that

$$
s_{P} \geq|P|\left(\operatorname{ch}\left(\theta_{1} /|P|\right)-1\right) \geq \frac{\theta_{1}^{2}}{2|P|} \geq \frac{c}{V},
$$

with $c=(\operatorname{arccosh} \sqrt{2})^{2} / 2$. The proof of Fact 2 is now complete with $a$ as above and $b=c / V$.

To show Fact 1 one proceeds essentially in the same way; only the energy bound is more easily derived in a slightly different way: again it suffices to consider three spins $n_{0}, n, n_{1}$ with the energy

$$
e(n)=n \cdot n_{0}+n \cdot n_{1},
$$

direct minimization over $n$ yields

$$
n=\lambda\left(n_{0}+n_{1}\right)
$$

with $\lambda$ fixed by the requirement $n^{2}=1$. So $n$ has to lie on the great circle connecting $n_{0}$ and $n_{1}$. As before we conclude from this that the minimal energy for a path is obtained by moving along the shorter part of a great circle (geodesic) connecting $n_{0}$ to $n_{1}$ in equal steps.

The corollary as well as the main result are obvious consequences.

Thus we have learned that the perturbative expansions of the compact ferromagnetic model and the noncompact model are related just by flipping the sign of $\beta$, even though doing the same sign flip to the full model leads to a completely different model - the antiferromagnet. 
The relation between the asymptotic expansions in $1 / \beta$ for the compact and noncompact models is given in (2.20). For the two point function this means for instance

$$
w_{-, 1}^{(n)}=(-1)^{n+1} w_{+, 1}^{(n)} .
$$

This should be contrasted with the relations between the perturbation expansions of the two-point function between the ferromagnetic and anti ferromagnetic compact models, which follows from $(2.13)$

$$
\left.w_{-, 1}^{(n)}(x, y)\right|_{\text {antiferro }}=\left.\epsilon_{x} \epsilon_{y} w_{-, 1}^{(n)}(x, y)\right|_{\text {ferro }} .
$$

It is instructive to see what this means for the lowest order (tree graph) asymptotics of the two point function:

$$
\begin{array}{ll}
\left\langle n_{x} \cdot n_{y}\right\rangle=1+\frac{1}{\beta} w_{+, 1}^{(0)}(x, y)+O\left(\beta^{-2}\right) & \text { ferromagnet } \\
\left\langle n_{x} \cdot n_{y}\right\rangle=\left(1+\frac{1}{\beta} w_{+, 1}^{(0)}(x, y)\right) \epsilon_{x} \epsilon_{y}+O\left(\beta^{-2}\right) & \text { antiferromagnet } \\
\left\langle n_{x} \cdot n_{y}\right\rangle=1-\frac{1}{\beta} w_{+, 1}^{(0)}(x, y)+O\left(\beta^{-2}\right) & \text { noncompact model }
\end{array}
$$

where

$$
w_{+, 1}^{(0)}(x, y)=\frac{N}{V} \sum_{p \neq 0} \frac{\cos p \cdot(x-y)-1}{E_{p}}
$$

with $E_{p}$ as in Eq. (3.22) below.

Note that although $W_{+}[H]$ for the ferromagnet and the antiferromagnet are related by flipping the sign of $\beta$, this is no longer true for the asymptotic expansions, even in finite volume. Rather the sign flipped perturbative expansion is that of the noncompact model.

\section{Dual formulations and large $N$ correspondence}

The goal of this section is to establish a relation between the large $N$ expansions of invariant correlators in the compact and the noncompact sigma-models. In the compact model the large $N$ expansion is based on the familiar dual formulation, which here has to be modified due to the fixed spin. In the noncompact model the duality transformation is ill defined but by performing Gaussian integrations in horospherical coordinates, one can obtain a well-defined counterpart of the dual formulation. 


\subsection{Noncompact generating functional via horospherical coordinates}

Before turning to the expansions proper we present here an exact rewriting of $W_{-}[H]$ based on a partial evaluation where the $\left(\operatorname{dim} \mathbb{H}_{N}\right)^{V}$ integrations are reduced to $V$ integrations. The point of departure is the fact that the hyperboloid $\mathbb{H}_{N}$ admits an alternative parameterization in terms of so-called horospherical coordinates. These arise naturally from the Iwasawa decomposition of $\mathrm{SO}_{0}(1, N)$. Here it suffices to note the relation to the hyperbolic spins

$$
n^{0}=\operatorname{ch} \theta+\frac{1}{2} \sum_{i=1}^{N-1} t_{i}^{2} e^{-\theta}, \quad n^{1}=\operatorname{sh} \theta+\frac{1}{2} \sum_{i=1}^{N-1} t_{i}^{2} e^{-\theta}, \quad n^{i}=e^{-\theta} t_{i-1}, \quad i=2, \ldots, N
$$

and that $\left(\theta, t_{1}, \ldots, t_{N-1}\right) \in \mathbb{R}^{N}$ defines a globally valid system of coordinates. It is convenient to write $\vec{t}=\left(t_{1}, \ldots, t_{N-1}\right)$ and $\vec{t} \cdot \overrightarrow{t^{\prime}}=t_{1} t_{1}^{\prime}+\ldots t_{N-1} t_{N-1}^{\prime}$. For the dot product of two spins $n_{x}, n_{y} \in \mathbb{H}_{N}$ this gives

$$
n_{x} \cdot n_{y}=\operatorname{ch}\left(\theta_{x}-\theta_{y}\right)+\frac{1}{2}\left(\overrightarrow{t_{x}}-\overrightarrow{t_{y}}\right)^{2} e^{-\theta_{x}-\theta_{y}},
$$

and for the measure in $(2.2)$

$$
d \Omega_{-}(n)=e^{-\theta(N-1)} d \theta d t_{1} \ldots d t_{N-1}=e^{-\theta(N-1)} d \theta d \vec{t} .
$$

The key advantage of horospherical coordinates is manifest from (3.1), (3.2): for a quadratic action of the form $S_{-}$in (2.1) the integrations over the $\vec{t}$ variables are Gaussian and can be performed without approximations. We refer to [4] for the derivation and only note the result

$$
\begin{aligned}
& \exp W_{-}[H]=\exp \left\{-\frac{1}{2} \sum_{x, y} H_{x y}\right\} \mathcal{N} \int_{\mathcal{D}(H)} \prod_{x \neq x_{0}} d a_{x} \\
& \times \exp \left\{-\frac{N+1}{2} \operatorname{Tr} \ln \widehat{A}-\frac{\beta}{2} \sum_{x \neq x_{0}} a_{x}+\frac{\beta}{2}\left(\widetilde{A}^{-1}\right)_{x_{0} x_{0}}^{-1}\right\} .
\end{aligned}
$$

Here

$$
A_{x y}=-\Delta_{x y}+\frac{1}{\beta} H_{x y}+\delta_{x y} a_{x}=\widetilde{A}_{x y}+a_{x_{0}} \delta_{x x_{0}} \delta_{x y},
$$

and $\widehat{A}_{x y}$ is the matrix obtained from $A_{x y}$ by omitting the $x_{0}$-th row and column. The domain $\mathcal{D}(H)$ is an algebraic variety described by

$$
\mathcal{D}(H)=\left\{a \in[-2 d, \infty]^{V-1} \mid \operatorname{det} \widehat{A}>0, \widehat{A} \text { positive semidefinite }\right\} .
$$


We also anticipate from [4] the following

Result: The correlation functions $W_{-, r}$ admit an asymptotic expansion of the form (2.10), whose (uniquely defined) expansion coefficients coincide with those defined by the Laplace expansion of $(3.4)$ where $\mathcal{D}(H)$ has been replaced by $\mathbb{R}^{V-1}$. In turn these coefficients coincide with those of the formal large $N$ expansion of

$$
\begin{aligned}
\exp W_{-}^{d}[H] & =\exp \left\{-\frac{1}{2} \sum_{x, y} H_{x y}\right\} \mathcal{N} \int \prod_{x \neq x_{0}} d \alpha_{x} \exp \left\{-(N+1) S_{-}[\alpha, H]\right\} \\
S_{-}[\alpha, H] & =\frac{1}{2} \operatorname{Tr} \ln \widehat{A}+i \sum_{x \neq x_{0}} \alpha_{x}-\frac{1}{2 \lambda}\left(\widetilde{A}^{-1}\right)_{x_{0} x_{0}}^{-1}
\end{aligned}
$$

where $a_{x}$ corresponds to $2 i \lambda \alpha_{x}$. However

$$
W_{-}[H] \neq W_{-}^{d}[H]
$$

Heuristically the origin of the 'dual' generating functional $W_{-}^{d}[H]$ can be understood by a dualization of the 'spatial' spin components $\vec{n}_{x}, x \in \Lambda$, and a formal contour deformation in field space [9]. The formal nature of the latter is responsible for (3.8), although the large $N$ expansion coefficients of $W_{-}[H]$ are correctly reproduced.

\subsection{Dual formulation: compact model}

In the compact model the counterpart of (3.7) is obtained along the familiar lines: one first implements the constraints $n_{x} \cdot n_{x}=1$ via a Lagrange multiplier field and then performs the Gaussian integrations. Of course in the compact model no gaugefixing is required and the result is well-known. For the purposes of comparing with the noncompact model, however, we want to perform the dualization here in the fixed spin gauge, i.e. for $W_{+}[H]$, in which case certain modifications occur. The resulting dual generating functional $W_{+}^{d}[H]$ is an exact rewriting of the original one: $W[H]=W_{+}[H]=$ $W_{+}^{d}[H]$. 
In the fixed spin gauge Gaussian integrals of the following form arise

$$
\begin{aligned}
& \int \prod_{x} d \phi_{x} \delta\left(\phi_{x_{0}}\right) \exp \left\{-\frac{1}{2} \sum_{x, y} \phi_{x} A_{x y} \phi_{y}+\sum_{x} J_{x} \phi_{x}\right\} \\
& =(2 \pi)^{\frac{V-1}{2}}(\operatorname{det} \widehat{A})^{-1 / 2} \exp \left\{\frac{1}{2} \sum_{x, y} J_{x}\left(\widehat{A}^{-1}\right)_{x y} J_{y}\right\} \\
& \int \prod_{x \neq x_{0}} d \phi_{x} \exp \left\{-\frac{1}{2} \sum_{x, y} \phi_{x} A_{x y} \phi_{y}\right\} \\
& =(2 \pi)^{\frac{V-1}{2}}(\operatorname{det} \widehat{A})^{-1 / 2} \exp \left\{-\frac{\phi_{x_{0}}^{2}}{2\left(A^{-1}\right)_{x_{0} x_{0}}}\right\},
\end{aligned}
$$

for a real field $\phi_{x}, x \in \Lambda$, and a symmetric invertible $L^{d} \times L^{d}$ matrix $A$, for which we assume that $\left(A^{-1}\right)_{x_{0} x_{0}} \neq 0$. Here $\widehat{A}$ is the $\left(L^{d}-1\right) \times\left(L^{d}-1\right)$ matrix arising from $A$ by deleting its $x_{0}$-th row and column. The inverse of $\widehat{A}$ can be expressed in terms of the inverse of $A$ via

$$
\left(\widehat{A}^{-1}\right)_{x y}=\left(A^{-1}\right)_{x y}-\frac{\left(A^{-1}\right)_{x x_{0}}\left(A^{-1}\right)_{y x_{0}}}{\left(A^{-1}\right)_{x_{0} x_{0}}} .
$$

This equation makes sense a priori for $x, y, \neq x_{0}$, but we may trivially extend the matrix $\widehat{A}^{-1}$ to a $L^{d} \times L^{d}$ matrix by setting $\left(\left[\widehat{A}^{-1}\right]_{\text {ext }}\right)_{x_{0} x}=0$ in accordance with Eq. (3.10). Then $A$ and $\left[\widehat{A}^{-1}\right]_{\text {ext }}$ are of course not inverse to each other

$$
\sum_{z} A_{x z}\left(\left[\widehat{A}^{-1}\right]_{\mathrm{ext}}\right)_{z y}=\delta_{x y}-\delta_{x x_{0}} \frac{\left(A^{-1}\right)_{x_{0} y}}{\left(A^{-1}\right)_{x_{0} x_{0}}}
$$

To verify (3.9) one writes $\sum_{x, y} \phi_{x} A_{x y} \phi_{y}=\sum_{x, y \neq x_{0}} \phi_{x} A_{x y} \phi_{y}+2 \phi_{x_{0}} \sum_{x \neq x_{0}} A_{x_{0} x} \phi_{x}+$ $\phi_{x_{0}}^{2} A_{x_{0} x_{0}}$. To get (3.9a) one first does the trivial $\phi_{x_{0}}$ integration. To get $(3.9 \mathrm{~b})$ one uses (3.11). The determinant of $\widehat{A}$ is related to that of $A$ by

$$
\operatorname{det} A=\frac{\operatorname{det} \widehat{A}}{\left(A^{-1}\right)_{x_{0} x_{0}}} .
$$

Often a term in the $x_{0}$-th matrix element on the diagonal of $A$ has to be split off according to $A_{x y}=\widetilde{A}_{x y}-c \delta_{x y} \delta_{x_{0} x}$. In this case the inverse of $A$ is related to the inverse of $\widetilde{A}$ by

$$
\left(A^{-1}\right)_{x y}=\left(\widetilde{A}^{-1}\right)_{x y}+\frac{c}{1-c\left(\widetilde{A}^{-1}\right)_{x_{0} x_{0}}}\left(\widetilde{A}^{-1}\right)_{x x_{0}}\left(\widetilde{A}^{-1}\right)_{y x_{0}}
$$


In particular $A_{x_{0} x_{0}}-\left(A^{-1}\right)_{x_{0} x_{0}}^{-1}=\widetilde{A}_{x_{0} x_{0}}-\left(\widetilde{A}^{-1}\right)_{x_{0} x_{0}}^{-1}$ and

$$
\frac{1}{\left(A^{-1}\right)_{x_{0} x_{0}}}=-c+\frac{1}{\left(\widetilde{A}^{-1}\right)_{x_{0} x_{0}}}, \quad \frac{\left(A^{-1}\right)_{x x_{0}}}{\left(A^{-1}\right)_{x_{0} x_{0}}}=\frac{\left(\widetilde{A}^{-1}\right)_{x x_{0}}}{\left(\widetilde{A}^{-1}\right)_{x_{0} x_{0}}}
$$

With these preparations at hand the dualization of $W_{+}[H]$ is straightforward. It is instructive to start from $W[H]$, single out one spin, $n_{x_{0}}$, and to postpone its dualization. Inserting

$$
\delta\left(n_{x}^{2}-1\right)=(N+1) \int \frac{d \alpha_{x}}{2 \pi} \exp \left\{-i(N+1) \alpha_{x}\left(n_{x}^{2}-1\right)\right\} \quad \text { for } \quad x \neq x_{0},
$$

the interchange in the order of integrations can be justified and the relevant Gaussian is of the form $(3.9 \mathrm{~b})$

$$
\begin{aligned}
& \int \prod_{x \neq x_{0}} d n_{x} \exp \left\{-\frac{1}{2} \frac{N+1}{\lambda} \sum_{x, y} n_{x} \widetilde{A}_{x y} n_{y}\right\} \\
& =\operatorname{Const}(\operatorname{det} \widehat{A})^{-(N+1) / 2} \exp \left\{-\frac{1}{2} \frac{N+1}{\lambda}\left(\widetilde{A}^{-1}\right)_{x_{0} x_{0}}^{-1} n_{x_{0}}^{2}\right\}, \\
& \widetilde{A}_{x y}=-\Delta_{x y}+2 i \lambda \alpha_{x}\left(1-\delta_{x_{0} x}\right) \delta_{x y}-\frac{\lambda}{N+1} H_{x y} .
\end{aligned}
$$

This gives for the dual generating functionals

$$
\begin{aligned}
\exp W^{d}[H] & =\int d n_{x_{0}} \delta\left(n_{x_{0}}^{2}-1\right) \exp W_{n_{x_{0}}}^{d}[H] \\
\exp W_{n_{x_{0}}}^{d}[H] & =\exp \left\{-\frac{1}{2} \sum_{x, y} H_{x y}\right\} \mathcal{N} \int \prod_{x \neq x_{0}} d \alpha_{x} \exp \left\{-(N+1) S_{n_{x_{0}}}[\alpha, H]\right\} \\
S_{n_{x_{0}}}[\alpha, H] & =\frac{1}{2} \operatorname{Tr} \ln \widehat{A}-i \sum_{x \neq x_{0}} \alpha_{x}+\frac{1}{2 \lambda} n_{x_{0}}^{2}\left(\widetilde{A}^{-1}\right)_{x_{0} x_{0}}^{-1} .
\end{aligned}
$$

Here we used the fact $\widehat{\widetilde{A}}=\widehat{A} ; W_{n_{x_{0}}}^{d}[H]$ is the generating functional in the fixed spin gauge and $S_{n_{x_{0}}}[\alpha, H]$ is its dual action. We shall also write $S_{+}[\alpha, H]$ for $S_{n^{\uparrow}}[\alpha, H]$ and $W_{+}^{d}[H]$ for $W_{n^{\uparrow}}^{d}[H]$. In contrast to the noncompact model one has

$$
W_{+}^{d}[H]=W_{+}[H], \quad W^{d}[H]=W[H]
$$

and also $W_{+}[H]=W[H]$, by $(2.4)$. 
As a check on (3.17) one can verify that by dualizing also the last spin one recovers the familiar expressions. Indeed,

$$
\begin{aligned}
& \int d n_{x_{0}} \delta\left(n_{x_{0}}^{2}-1\right) \exp \left\{-\frac{(N+1)}{2 \lambda}\left(\widetilde{A}^{-1}\right)_{x_{0} x_{0}}^{-1} n_{x_{0}}^{2}\right\} \\
& =\left(\frac{2 \pi \lambda}{N+1}\right)^{(N-1) / 2} \int d \alpha_{x_{0}} e^{i(N+1) \alpha_{x_{0}}}\left[\left(\widetilde{A}^{-1}\right)_{x_{0} x_{0}}^{-1}+2 i \lambda \alpha_{x_{0}}\right]^{-(N+1) / 2} .
\end{aligned}
$$

Using now (3.13) for $c=-2 i \lambda \alpha_{x_{0}}$ and (3.12) one obtains

$$
\begin{aligned}
\exp W[H] & =\mathcal{N} \int \prod_{x} d \alpha_{x} \exp \left\{-\frac{N+1}{2} \operatorname{Tr} \ln A+i \sum_{x} \alpha_{x}\right\}, \\
A_{x y} & =-\Delta_{x y}+2 i \lambda \alpha_{x} \delta_{x y}-\frac{\lambda}{N+1} H_{x y},
\end{aligned}
$$

as required.

\subsection{Finite volume mass gap and basic propagators}

The saddle point expansions of $W_{ \pm}^{d}[H]$ define to leading order the gap equations and the invariant two-point functions. In both quantities the dependence on the site $x_{0}$ of the fixed spin drops out, see section 3.6. The result for the leading order two-point function can be written in the form

$$
\lambda W_{ \pm, 1}^{(0)}(x, y)= \pm \lambda D_{ \pm}(x-y)-1=\left.\left\langle n_{x} \cdot n_{y}\right\rangle\right|_{N=\infty}-1
$$

where $D_{ \pm}(x-y)$ is the basic propagator. For the compact model it has the well-known structure $D_{+}(x)=\left.D(x)\right|_{\omega=\omega_{+}}$, where

$$
D(x)=\frac{1}{V} \sum_{p} \frac{e^{i p \cdot x}}{E_{p}+\omega}
$$

with the sum over all $p=\frac{2 \pi}{L}\left(n_{1}, \ldots, n_{d}\right), n_{i}=0,1, \ldots, L-1$, and $E_{p}:=2 d-2 \sum_{\mu} \cos (p$. $\hat{\mu})$. Further $\omega_{+}=\omega_{+}(\lambda, V)$ is the dynamically generated finite volume mass ${ }^{2}$ term determined by the gap equation $D(0)=1 / \lambda$. A subtlety is due to the fact that the equation for $\omega_{+}(\lambda, V)$ is an algebraic equation of degree $O(V)$ and therefore has $O(V)$ solutions. Among those solutions there is always a unique positive one, but it is not obvious that this is always the physically relevant one: in dimension $d \geq 3$ for weak coupling there is spontaneous symmetry breaking and it has been found in $[14,15]$ that at least in the determination of the constrained effective potential one has to take a negative solution of the gap equation. Here we want to eliminate these complications by always working in the 'strong coupling' regime, which we will now specify. 
In the infinite volume limit the gap equation becomes

$$
1=\lambda \int \frac{d^{d} p}{(2 \pi)^{d}} \frac{1}{E_{p}+\omega},
$$

and it has no negative solutions. It has a unique positive solution for $\lambda \geq \lambda_{*}(d)$, where $\lambda_{*}(2)=0$ and $\lambda_{*}(d)>0$ for $d \geq 3\left(\lambda_{*}(d)\right.$ is known as the critical coupling of the spherical model). From now on we will assume $\lambda>\lambda_{*}(d)$ and always require $\omega_{+}(\lambda, V)>0$. In this case it is possible to show that the finite volume corrections $\omega_{+}(\lambda, V)-\omega_{+}(\lambda, \infty)$ are exponentially small.

In the noncompact model one has similarly $D_{-}(x)=\left.D(x)\right|_{\omega=\omega_{-}}$, where $\omega_{-}$is a solution of the gap equation $\lambda D(0)=-1$, see Eqs. (3.54), (3.56) in section 3.6. Clearly analytically continuing the multivalued algebraic function $\omega_{+}(-\lambda, V)$ to negative values of $\lambda$ and setting $\omega_{-}(\lambda, V):=\omega_{+}(-\lambda, V)$ will produce solutions of this gap equation. But again there are $O(V)$ such solutions, all of which are negative. To see this it is convenient to split off the zero momentum mode and to write

$$
D(0)=\frac{1}{V \omega}+f(\omega), \quad f(\omega)=\frac{1}{L^{d}} \sum_{0 \neq n \in[0, L-1]^{d}} \frac{1}{\omega+\omega_{n}}
$$

where $\omega_{n}=E_{2 \pi n / L}, n=\left(n_{1}, \ldots, n_{d}\right)$. Here $f(\omega)$ is a meromorphic functions with simple poles along the negative real axis. The first pole is at $\omega=-4 \sin ^{2}(\pi / L)$, the others are at approximately integer multiples thereof. Specifically

$$
\omega_{n}=-4(\pi / L)^{2}\left(n_{1}^{2}+\ldots+n_{d}^{2}\right)+O\left(1 / L^{4}\right) .
$$

In terms of the function $f$ the gap equations $\pm \lambda D(0)=1$ read

$$
\lambda f(w)= \pm 1-\frac{\lambda}{L^{d} \omega}
$$

In the compact model (upper sign) is has a unique solution $\omega_{+}(\lambda, L)>0$ for any given $\lambda>\lambda_{*}(d)$; all the other $O(V)$ solutions are negative. In the noncompact case there are only $O(V)$ negative solutions, one in each interval of the $\omega$ axis between consecutive poles of $f$.

To obtain an asymptotic expansion in $1 / N$ one has to pick the solution corresponding to the absolute maximum of the dual Boltzmann factor. But there is a simpler way to do this selection: if the large $N$ expansion is to yield an asymptotic expansion of the invariant two-point function, we have to demand that $-\lambda D(x) \geq 1$ for all $x$. Since $\left\langle n_{x} \cdot n_{y}\right\rangle \geq 1$ in the noncompact model, this is a necessary condition for the interpretation 
of $D_{-}(x-y)=\left.D(x-y)\right|_{\omega=\omega_{-}}$as the leading order two-point function. This condition fixes the solution to lie in the interval $\omega \in\left(-4 \sin ^{2} \pi / L, 0\right)$. Indeed, $-\lambda D(x) \geq 1$ implies

$$
\begin{array}{r}
\sum_{x}(1-\cos q \cdot x)(-\lambda D(x)) \geq V, \quad q \neq 0, \\
\Longleftrightarrow \frac{1}{-\omega V} \frac{1}{1+\omega / E_{q}} \geq \frac{1}{\lambda}, \quad q \neq 0 .
\end{array}
$$

Since $\omega<0$ both factors on the left hand side must be positive. Hence $E_{q}+\omega>0$ for all $q \neq 0$, for which $\omega>-4 \sin ^{2} \pi / L$ is a necessary and sufficient condition.

It is easy to sharpen this bound to

$$
\omega>-\frac{4}{2 d+1} \sin ^{2} \frac{\pi}{L}
$$

by noting that

$$
0>-\frac{V}{\lambda}=\sum_{p} \frac{1}{E_{p}+\omega} \geq \frac{1}{\omega}+\frac{2 d}{4 \sin ^{2} \frac{\pi}{L}+\omega} .
$$

From now on we write $\omega_{-}(\lambda, V) \in\left(-4 \sin ^{2} \pi / L, 0\right)$ for the unique root of the $\lambda D(0)=-1$ gap equation such that $-\lambda D_{-}(x) \geq 1$ for all $x$. Its large volume asymptotics comes out as

$$
-V \omega_{-}(\lambda, V)= \begin{cases}\frac{4 \pi}{\ln V}(1+O(1 / \ln V)) & d=2, \\ \left(\frac{1}{\lambda}+C_{d}\right)^{-1}+O\left(V^{-\frac{d-2}{d}}\right) & d \geq 3 .\end{cases}
$$

where $C_{d}=\int_{0}^{2 \pi} \frac{d^{d} p}{(2 \pi)^{d}} \frac{1}{E_{p}}$.

In summary, to leading order the invariant spin two-point functions in the compact ferromagnetic and in the noncompact model can be written in the form (3.21), where

$$
D_{ \pm}(x):=\left.D(x)\right|_{\omega=\omega_{ \pm}(\lambda, V)}, \quad \text { with } \quad \omega_{ \pm}(\lambda, V) \text { solution of } \lambda D(0)= \pm 1
$$

In both the compact model and the noncompact model there is a unique root of the gap equation $\lambda D(0)= \pm 1$ such that $\lambda D_{ \pm}(x) \leq \pm 1$ for all $x$. From now on the symbols $D_{ \pm}(x)$ will always refer to the unique propagators with this property. They satisfy

$$
\begin{aligned}
& 1 \leq-\lambda D_{-}(x)<-1-\frac{2 \lambda}{V \omega_{-}} \\
& -1+\frac{2 \lambda}{V \omega_{+}}<\lambda D_{+}(x) \leq 1 .
\end{aligned}
$$

The $\omega_{ \pm}$dependent bounds follows from $D(x)>2 /(V \omega)-D(0)$. In particular $0<$ $-\omega_{-}<\lambda / V$. 
For $V \rightarrow \infty, D_{+}(x)$ approaches the massive free propagator of squared mass $\omega_{+}(\lambda, \infty)$, while $D_{-}(x)$ becomes massless and naively appears to be ill-defined. If one inserts, however, for $\omega$ the $L$-dependent solution of the gap equation $-\lambda D_{-}(0)=1$ one can rewrite $\lambda D_{-}(x)$ in such a way that the existence of the thermodynamic limit is manifest:

$$
\left.\left\langle n_{x} \cdot n_{y}\right\rangle\right|_{N=\infty}=-\lambda D_{-}(x-y)=1+\frac{\lambda}{V} \sum_{k \neq 0} \frac{1-\cos k \cdot(x-y)}{E_{k}+\omega_{-}},
$$

which for $V \rightarrow \infty$ has the well-defined limit

$$
1+\lambda \int \frac{d^{d} k}{(2 \pi)^{d}} \frac{1-\cos k \cdot(x-y)}{E_{k}} .
$$

\subsection{Large $N$ correspondence}

The results of sections 2 and 3 now lead to the

Result: Let $W_{r,+}$ and $W_{r,-}$ denote the connected parts of the invariant $2 r$-point functions $\left\langle n_{x_{1}} \cdot n_{y_{1}} \ldots n_{x_{r}} \cdot n_{y_{r}}\right\rangle$ in the $\mathrm{SO}(N+1)$ and in the $\mathrm{SO}(1, N)$ nonlinear sigma-model, respectively. (With bare coupling $\beta$, defined on a hypercubic lattice $\Lambda \subset \mathbb{Z}^{d}$ of volume $L^{d}$ with periodic boundary conditions, and a suitable gauge fixing in the noncompact case.) Then the $W_{ \pm, r}$ have well-defined "large $N$ " asymptotic expansions of the form (2.10), with $\lambda:=(N+1) / \beta$ fixed. Further:

(a) The coefficient functions $W_{ \pm, r}$ are translation invariant. The lowest order two-point functions have the form $W_{ \pm, 1}^{(0)}(x, y)= \pm D_{ \pm}(x, y)-1 / \lambda$, where $D_{ \pm}(x)=\left.D(x)\right|_{\omega \rightarrow \omega_{ \pm}}$. Here $D(x)$ is the free propagator of squared mass $\omega$ and $\omega_{ \pm}(\lambda, V)$ are the unique solutions of the gap equations $\pm \lambda D(0)=1$ with the property $\pm \lambda D(x) \geq 1$, for all $x$.

(b) For all $r \geq 1, s \geq 0$, there exist unique functionals $X_{r}^{(s)}[D](\lambda)$ of a free propagator $D$ such that $W_{+, r}^{(s)}=X_{r}^{(s)}\left[D_{+}\right](\lambda)$ are the coefficients in the compact model and $W_{-, r}^{(s)}=(-1)^{r} X_{r}^{(s)}\left[D_{-}\right](-\lambda)$ are the coefficients in the noncompact model.

Proof. The existence of the large $N$ asymptotic expansion has been shown in the compact model in [16], for $W[H]$ (or its the counterpart with a linear source coupling). Since $W_{+}^{d}[H]=W^{d}[H]=W[H]$, this directly carries over to $W_{+}[H]$. In the noncompact model the result has been anticipated in Subsection 3.1; for the derivation we refer to the forthcoming paper [4]. The two statements in part (a) follow from sections 2.1 and 3.3, respectively. For (b) we use the result stated in section 3.1, namely that $W_{-}^{d}[H]$ (although not equivalent to $W_{-}[H]$ and not obtainable from a well-defined duality transformation) 
generates the correct large $N$ asymptotic expansion. Given this, it suffices to note that $W_{+}^{d}[H]$ in (3.17) and $W_{-}^{d}[H]$ in (3.7) have the same structure with actions that are related by the involution

$$
S_{+}[\alpha, H] \mapsto S_{-}[\alpha, H] \quad \text { for } \quad \alpha_{x} \mapsto-\alpha_{x}, \quad \lambda \mapsto-\lambda
$$

Hence, if indeed the moments of $W_{+}^{d}[H]$ and $W_{-}^{d}[H]$ have asymptotic expansions of the form (2.10), their coefficients are uniquely defined and must therefore also be related by the involution (3.35).

For the sake of clarity let us add two remarks:

First, $W_{+}[H]$ and $W_{-}[H]$ do not coincide exactly, as follows from (3.8), (3.18) and the fact that the boundary $\mathcal{D}(H)$ in (3.6) is invariant under the involution (3.35). In physics terms this amounts to the observation made before that the antiferromagnet and the noncompact model are inequivalent.

Second, (b) does not state that the large $N$ coefficients of the compact (ferromagnetic or antiferromagnetic) model and the noncompact model are related by flipping the sign of $\lambda$. The latter is certainly incorrect. Rather the dependence on $D_{ \pm}$is same after flipping the sign of $\lambda$, but $D_{+}$and $D_{-}$are very different and independently defined functions of the lattice distance. So, given the large $N$ coefficients of $W_{+, r}$, as a function of the lattice points, there would be no simple way to construct the large $N$ expansion of $W_{-, r}$.

\subsection{Schwinger-Dyson equations}

It is instructive to look at the previous result from the viewpoint of the Schwinger-Dyson (SD) equations. Under the assumption that asymptotic expansions of $W_{-, r}$ and $W_{+, r}$ are known to exist, this also provides an alternative derivation of the result.

In the compact model the Schwinger-Dyson equations for the moments of $W[H]$ have, to our knowledge, first been formulated by M. Lüscher [17]. In the noncompact model the necessity to gauge fix requires modifications. In the fixed spin gauge the modifications in the equations are minimal, but they inevitably refer to the preferred point $x_{0}$. In the following we formulate the SD equations in parallel for the gauge fixed functionals $W_{ \pm}[H]$.

The result is:

$$
\begin{aligned}
& \pm\left.\beta \Delta_{z}\left[h_{z y} W_{ \pm}-h_{z x} W_{ \pm}-h_{z x} h_{x y} W_{ \pm}-\left(h_{z x} W_{ \pm}\right)\left(h_{x y} W_{ \pm}\right)\right]\right|_{z=x} \\
& +\sum_{z \neq x} H_{x z}\left[h_{z y} W_{ \pm}-h_{z x} W_{ \pm}-h_{x y} W_{ \pm}-h_{x z} h_{x y} W_{ \pm}-\left(h_{z x} W_{ \pm}\right)\left(h_{x y} W_{ \pm}\right)\right] \\
& -N\left(1-\delta_{x y}\right)\left(h_{x y} W_{ \pm}+1\right)=0 .
\end{aligned}
$$


Again the upper sign refers to the compact model and the lower one to the noncompact model. In addition there is the following minor but essential modification: in the compact case (3.36) holds for all $x, y \in \Lambda$, since $W_{+}[H]$ coincides identically with the generating functional $W[H]$ defined without gauge fixing, see (2.4). In contrast, in the noncompact case it holds for all $x_{0} \neq x \in \Lambda$ and all $y \in \Lambda$. Specializing to $y=x_{0}$ thus results in an equation that is qualitatively different from the others.

The derivation of (3.36) is based on the invariance of the product measure $\prod_{x} d \Omega\left(n_{x}\right)$ under rotation of any one of the spins, $n_{x_{1}}$, say, where $x_{1} \neq x_{0}$ in the noncompact model. For an infinitesimal rotation with the Lie algebra element $t^{a b}$ we write

$$
\delta_{x_{1}}^{a b} n_{x}^{d}=\delta_{x_{1} x} n_{x}^{c}\left(t^{a b}\right)_{c}^{d}
$$

For later use we pick an explicit basis and note the completeness relations:

$$
\begin{array}{ll}
s o(N+1): \quad & \left(t^{a b}\right)_{c}{ }^{d}=\delta_{c}^{a} \delta^{b d}-\delta_{c}{ }^{b} \delta^{a d}=\left(t_{a b}\right)_{c}{ }^{d}, \\
& \frac{1}{2} \sum_{a b}\left(t^{a b}\right)_{c}{ }^{d}\left(t_{a b}\right)_{e}{ }^{f}=\delta_{c e} \delta^{f d}-\delta_{c}{ }^{f} \delta_{e}{ }^{d}, \\
s o(1, N): \quad & \left(t^{a b}\right)_{c}{ }^{d}=\delta_{c}^{a} \eta^{b d}-\delta_{c}{ }^{b} \eta^{a d}=\eta^{a a^{\prime}} \eta^{b b^{\prime}}\left(t_{a^{\prime} b^{\prime}}\right)_{c}{ }^{d} \\
& \frac{1}{2} \sum_{a b}\left(t^{a b}\right)_{c}{ }^{d}\left(t_{a b}\right)_{e}{ }^{f}=\eta_{c e} \eta^{f d}-\delta_{c}{ }^{f} \delta_{e}{ }^{d},
\end{array}
$$

where $\eta$ is defined by $a^{c} \eta_{c d} b^{d}=a^{0} b^{0}-a^{1} b^{1}-\ldots a^{N} b^{N}$. Denoting by \langle\rangle$_{H}$ the source dependent averages this gives in a first step

$$
\left\langle-\left(\delta_{x}^{a b} S\right) \mathcal{O}+\sum_{z} H_{x z} t^{a b} n_{x} \cdot n_{z} \mathcal{O}+\delta_{x}^{a b} \mathcal{O}\right\rangle_{H}=0
$$

for any local function $\mathcal{O}=\mathcal{O}(\{n\})$ of the spins, and $x \neq x_{0}$ in the noncompact case. Specializing to $\mathcal{O}=n_{x} \cdot t^{a b} n_{y}$ and summing over $a, b$, using the completeness relations (3.38) gives

$$
\begin{aligned}
& \left\langle \pm\left.\beta \Delta_{z}\left[n_{z} \cdot n_{y}-n_{z} \cdot n_{x} n_{y} \cdot n_{x}\right]\right|_{z=x}+\sum_{z \neq x} H_{x z}\left[n_{z} \cdot n_{y}-n_{z} \cdot n_{x} n_{y} \cdot n_{x}\right]\right. \\
& \left.\quad-N\left(1-\delta_{x y}\right) n_{y} \cdot n_{x}\right\rangle_{H}=0 .
\end{aligned}
$$

Replacing the \langle\rangle$_{H}$ averages by functional derivatives of $W_{ \pm}[H]$ results in (3.36).

Coupled bilinear equations for the $W_{r}$ functions arise by differentiating (3.36) repeatedly and setting $H$ equal to zero. The equation obtained by $s$-fold differentiation involves all 
$W_{r}$ functions with $r \leq s+2$; as a consequence no finite subsystem of equations arises for any individual $W_{r}$.

Before turning to the large $\mathrm{N}$ expansion of the system (3.36) we wish to stress that even the exact SD equations do not determine their solution uniquely. To see this, let us momentarily denote the functional equations (3.36) with the upper and the lower sign by $(\mathrm{SD})_{+}$and $(\mathrm{SD})_{-}$, respectively. Further we write $W_{+, \beta}, W_{+,-\beta}$, and $W_{-, \beta}, \beta>0$, for the generating functionals of the ferromagnet, the anti-ferromagnet, and the noncompact model, respectively. Then, by construction, $W_{+, \beta}$ solves $(\mathrm{SD})_{+}$while both $W_{+,-\beta}$ and $W_{-, \beta}$ solve $(\mathrm{SD})_{-}$. (Consistency requires that substituting the rhs of (2.14) into the $(\mathrm{SD})_{-}$equation converts it back into a $(\mathrm{SD})_{+}$equation for $W_{+, \beta}$ evaluated on $H^{\epsilon}$. Using $(\Delta \epsilon f)_{x}=-\epsilon_{x}(\Delta f)_{x}-4 d \epsilon_{x} f_{x}$, this can be verified to be the case.) Thus if the exact SD equations were to determine their solution uniquely, $W_{+,-\beta}$ and $W_{-, \beta}$ would have to coincide. This however contradicts the discussion in section 2.2 , viz the antiferromagnet and the noncompact model are physically and mathematically inequivalent. The upshot is that somehow initial or boundary conditions have to be imposed on (3.36) to specify a solution uniquely. Since the exact equations couple all multipoint functions, this seems difficult to do concretely.

In contrast, the large $N$ ansatz (2.10) effectively converts the equations into ones which can be solved recursively, and 'initial' conditions can be specified. The structure of the large $N$ expanded SD equations is best explained by spelling out the first few (see [9]) from which one can read off the recursion pattern for the $W_{r}^{(s)}, r+s>1$, functions:

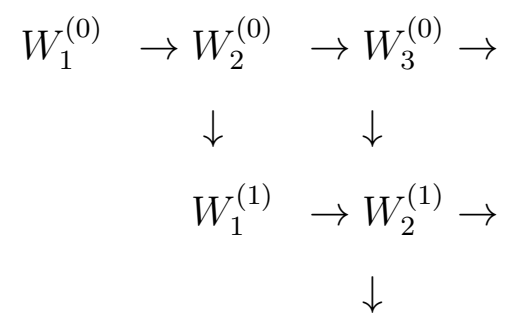

Fig. 1: Recursion pattern for the solution of the large $N$ expanded SD equations.

To compute a given coefficient all quantities having arrows pointing towards it are needed. The detailed form of the equations and the solutions is not essential for the following argument.

We only need: (i) the fact that the equation for $W_{1}^{(0)}$ is autonomous and reads

$$
\begin{aligned}
& \pm\left.\left.\Delta_{z}\left[W_{1}^{(0)}(z, y)-W_{1}^{(0)}(z, x)\right]\right|_{z=x} \mp \lambda W_{1}^{(0)}(x, y) \Delta_{z} W_{1}^{(0)}(z, x)\right|_{z=x} \\
& =1-\delta_{x y}+\lambda W_{1}^{(0)}(x, y)
\end{aligned}
$$

and (ii) the assumption that each recursion step in Fig. 1 has a unique solution. We first present the solutions of (3.41) and then discuss the status of the assumption (ii). 
A solution of (3.41) with the upper sign is

$$
W_{1}^{(0)}(x, y)=D_{+}(x-y)-1 / \lambda \quad \text { ferromagnet }
$$

Two solutions of (3.41) with the lower sign are

$$
\begin{array}{ll}
W_{1}^{(0)}(x, y)=\epsilon_{x} \epsilon_{y} D_{+}(x-y)-1 / \lambda & \text { anti-ferromagnet } \\
W_{1}^{(0)}(x, y)=-D_{-}(x-y)-1 / \lambda & \text { noncompact model }
\end{array}
$$

where for the verification of the first solution $(\Delta \epsilon f)_{x}=-\epsilon_{x}(\Delta f)_{x}-4 d \epsilon_{x} f_{x}$ has been used. For each solution $W_{1}^{(0)}(x, x)=0$ amounts to the corresponding gap equation. As discussed in section 3.3 the gap equations have $O(V)$ solutions other than the physical ones $\omega_{ \pm}$entering $D_{ \pm}$. Each solution of the gap equation defines a different solution of (3.41) via (3.42), (3.43), and in principle there could be others. The selection of a specific solution - here $D_{ \pm}$- can be justified either from the functional integral (in that it gives rise to valid asymptotic expansion) or from the physics one seeks to describe.

The uniqueness of the recursion (ii), to all orders, is probably difficult to establish directly from the SD equations. In a perturbative expansion of the $W_{r}$ the problem becomes linear and the fact that the perturbative expansions of the $W_{r}$ is uniquely determined by the equations has been pointed out by M. Lüscher [17]. In other words a potential nonuniqueness is known to be of order $O\left(\beta^{-\infty}\right)$, directly from the SD equations.

An indirect way to establish the uniqueness (ii) is by showing that the moments of the generating functionals $W_{ \pm}[H]$ have well-defined asymptotic expansions of the form (2.10). The expansion coefficients then must be unique, and since $W_{ \pm}[H]$ are solutions of (3.36) the coefficients $W_{ \pm, r}^{(s)}$ must be solutions of the expanded SD equations, and the unique solutions. This also implies that the solutions must be translation invariant, despite the preferred role of the $x=x_{0}$ equation. As mentioned earlier, the fact that the moments of $W_{+}[H]$ have an asymptotic expansion follows from [16]; for $W_{-}[H]$ this will be shown in [4]

Given the uniqueness of the recursion one can readily re-derive the result of section 3.4. From (3.36) and (2.10) it is clear that the involution

$$
\lambda \mapsto-\lambda, \quad W_{r}^{(n)}(\lambda) \mapsto(-)^{r} W_{r}^{(n)}(-\lambda)
$$

will map the Schwinger-Dyson equations of the compact model onto those of the noncompact model, to all orders of their large $N$ expansions. To draw conclusions about the solutions, however, the uniqueness asserted in (ii) is crucial. It implies that a sequence of solutions $W_{r}^{(n)}(\lambda)$ in the compact model, based on a specific choice of $W_{1,+}^{(0)}(\lambda)$, has a unique counterpart in the noncompact model based on the flipped initial solution $-W_{1,-}^{(0)}(-\lambda)$ of the lower sign SD equations (3.41). Since the physical solutions 
of the compact ferromagnet and the noncompact model are related in this way it follows that there exists unique functionals $X_{r}^{(s)}[D](\lambda)$ such that $W_{+, r}^{(s)}=X_{r}^{(s)}\left[D_{+}\right](\lambda)$ and $W_{-, r}^{(s)}=(-1)^{r} X_{r}^{(s)}\left[D_{-}\right](-\lambda)$, where $D_{ \pm}(x-y):= \pm\left(W_{ \pm, 1}^{(0)}(x, y)+1 / \lambda\right)$ are the physical solutions of (3.41). In summary, the previous discussion reproduces the parts (a),(b) of the result in section 3.4 .

We add some remarks. First, the involution (3.44) is the counterpart of (3.35). Let us repeat that the involutions (3.35) or (3.44) do not imply a simple relation between the $W_{r}^{(n)}(\lambda)$ and $W_{r}^{(n)}(-\lambda)$ as functions of the lattice points, because already $W_{1}^{(0)}(\lambda)$ and $W_{1}^{(0)}(-\lambda)$ are based on solutions of different gap equations. It is rather the functional dependence on $D_{ \pm}$which is the same.

The involution also does not map the large $N$ coefficients of the compact model into those of the antiferromagnet, defined by $\left.W_{+}[H]\right|_{\lambda \rightarrow-\lambda}$. The reason is that the large $N$ saddle point relevant for the antiferromagnet is not the one obtained by reversing the sign of $\lambda$ in the gap equation. Rather is is given by the same equation as the saddle point for the ferromagnet, as is manifest from (3.43). Generally the large $N$ coefficients of the antiferromagnet are obtained simply by introducing appropriate products of the alternating sign function $\epsilon_{x}$ in those of the ferromagnet, as explained in Section 2.2 .

The special role of the SD equations (3.36) for $y=x_{0}$ is related to the discussion in the paragraph following Eq. (2.8). It may suffice to illustrate the point with the lowest order equation (3.41). The solution of the $y=x_{0}$ equation in (3.41) is

$$
\left\langle n_{x}^{0}\right\rangle_{f . s .}=\bar{n}_{x}+O\left(\frac{1}{N+1}\right) \quad \text { with } \quad \bar{n}_{x}:=1+\lambda W_{1}^{(0)}\left(x_{0}, x\right)
$$

The interpretation of this solution as the nonzero average of the $n_{x}^{0}$ component follows from [1]. To higher orders the solutions of the $y=x_{0}$ equations produce corrections to (3.45) or non invariant three-point functions, etc.

\subsection{Algorithm in fixed spin gauge}

Here we illustrate the main results from sections 3.1 (existence of the asymptotic expansion) and 3.4 (compact-non-compact correspondence) by an explicit computation of the two- and four-point functions in the noncompact model to next-to-leading order. The starting point is the generating functional (3.5), where according to the result described, $\mathcal{D}(H)$ has been replaced by $\mathbb{R}^{V-1}$. Due to the gauge fixing a number of complications arise compared to the conventional large $N$ computations; in particular it is not obvious how translation invariant correlation functions are recovered.

Initially we keep the putative saddle point configuration $\omega_{x}, x \neq x_{0}$, generic and spe- 
cialize to $\omega_{x}=\omega_{-}$only later. We thus write

$$
a_{x}=\omega_{x}+\frac{u_{x}}{\sqrt{N+1}}, \quad u_{x} \in \mathbb{R}, \quad x \neq x_{0} .
$$

Although notationally cumbersome it is important to carefully distinguish "hatted", "tilde" and "plain" matrices. We set

$$
\begin{aligned}
& M_{x y}:=-\Delta_{x y}+\omega_{x} \delta_{x y}=: \widetilde{M}_{x y}+\omega_{x_{0}} \delta_{x x_{0}} \delta_{x y}, \quad \widetilde{D}_{x y}:=\left(\widetilde{M}^{-1}\right)_{x y} . \\
& U_{x y}:=u_{x} \delta_{x y}=: \widetilde{U}_{x y}+u_{x_{0}} \delta_{x x_{0}} \delta_{x y} .
\end{aligned}
$$

The matrices $M$ and $U$ are also defined for $x, y=x_{0}$. For $M$ we assume $\omega_{x_{0}} \neq \omega_{-}+\lambda$, which ensures its invertibility; in $U_{x y}$ the variable $u_{x_{0}}$ is non-dynamical. Further we write $\widehat{M}, \widehat{U}$ for the matrices obtained from $M, U$, respectively, by deleting the $x_{0}$-th row and column and put $\widehat{D}=\widehat{M}^{-1}$. Then

$$
\begin{gathered}
\widetilde{A}=\widetilde{M}+\frac{1}{\sqrt{N+1}} \widetilde{U}+\frac{1}{N+1} \lambda H, \\
\widetilde{A}^{-1}=\left(\mathbb{1}+\frac{1}{\sqrt{N+1}} \widetilde{D} \widetilde{U}+\frac{1}{N+1} \lambda \widetilde{D} H\right)^{-1} \widetilde{D}, \\
\widehat{A}=\widehat{M}+\frac{1}{\sqrt{N+1}} \widehat{U}+\frac{1}{N+1} \lambda \widehat{H}, \\
\ln \widehat{A}=\ln \widehat{M}+\ln \left(\mathbb{1}+\frac{1}{\sqrt{N+1}} \widehat{D} \widehat{U}+\frac{1}{N+1} \lambda \widehat{D} \widehat{H}\right) .
\end{gathered}
$$

The required expansions are for square matrices $a, b$

$$
\begin{aligned}
& \left(\mathbb{1}+\frac{a}{\sqrt{N+1}}+\frac{b}{N+1}\right)^{-1}=\mathbb{1}+\sum_{l \geq 1} \frac{1}{(N+1)^{l / 2}} q_{l}(a, b), \\
& q_{1}(a, b)=-a, \quad q_{2}(a, b)=a^{2}-b, \quad q_{3}(a, b)=-a^{3}+a b+b a, \\
& q_{4}(a, b)=a^{4}-a^{2} b-a b a-b a^{2}+b^{2}, \\
& \ln \left(\mathbb{1}+\frac{a}{\sqrt{N+1}}+\frac{b}{N+1}\right)=\sum_{l \geq 1} \frac{1}{(N+1)^{l / 2}} p_{l}(a, b), \\
& p_{1}(a, b)=a, \quad p_{2}(a, b)=-\frac{1}{2} a^{2}+b, \quad p_{3}(a, b)=\frac{1}{3} a^{3}-\frac{1}{2}(a b+b a), \\
& p_{4}(a, b)=-\frac{1}{4} a^{4}+\frac{1}{3}\left(b a^{2}+a b a+a^{2} b\right)-\frac{1}{2} b^{2} .
\end{aligned}
$$


Explicit formulas for the polynomials $q_{l}, p_{l}, l \geq 5$, can easily be found but will not be needed. With this notation one has

$$
\begin{aligned}
\left(\widetilde{A}^{-1}\right)_{x_{0} x_{0}} & =\widetilde{D}_{x_{0} x_{0}}\left[1+\sum_{l \geq 1} \frac{1}{(N+1)^{l / 2}} \widetilde{D}_{x_{0} x_{0}} Q_{l}\right], \\
Q_{l} & :=\widetilde{D}_{x_{0} x_{0}}^{-2}\left(q_{l}(\widetilde{D} \widetilde{U}, \lambda \widetilde{D} H) \widetilde{D}\right)_{x_{0} x_{0}}, \\
\ln \widehat{A} & =\ln \widehat{M}+\sum_{l \geq 1} \frac{1}{(N+1)^{l / 2}} p_{l}(\widehat{D} \widehat{U}, \lambda \widehat{D} \widehat{H}),
\end{aligned}
$$

from which the expansion of $S[a, H]$, i.e.

$$
\begin{aligned}
& S[a, H]=\frac{1}{2} \operatorname{Tr} \ln \widehat{A}+\frac{1}{2 \lambda} \sum_{x \neq x_{0}} a_{x}-\frac{1}{2 \lambda}\left(\widetilde{A}^{-1}\right)_{x_{0} x_{0}}^{-1}, \\
& S[a, H]=\sum_{l \geq 0} \frac{1}{(N+1)^{l / 2}} S_{l}[u, H]
\end{aligned}
$$

can readily be computed to any desired order. We present $S_{1}, \ldots, S_{4}$ first in the condensed form arising from (3.49), (3.50).

$$
\begin{aligned}
& S_{1}[u, H]=\frac{1}{2} \operatorname{Tr} p_{1}(\widehat{D} \widehat{U}, \lambda \widehat{D} \widehat{H})+\frac{1}{2 \lambda} \sum_{x \neq x_{0}} u_{x}+\frac{1}{2 \lambda} Q_{1} \\
& S_{2}[u, H]=\frac{1}{2} \operatorname{Tr} p_{2}(\widehat{D} \widehat{U}, \lambda \widehat{D} \widehat{H})+\frac{1}{2 \lambda}\left(Q_{2}-\widetilde{D}_{x_{0} x_{0}} Q_{1}^{2}\right) \\
& S_{3}[u, H]=\frac{1}{2} \operatorname{Tr} p_{3}(\widehat{D} \widehat{U}, \lambda \widehat{D} \widehat{H})+\frac{1}{2 \lambda}\left(Q_{3}-\widetilde{D}_{x_{0} x_{0}} 2 Q_{2} Q_{1}+\widetilde{D}_{x_{0} x_{0}}^{2} Q_{1}^{3}\right) \\
& S_{4}[u, H]=\frac{1}{2} \operatorname{Tr} p_{4}(\widehat{D} \widehat{U}, \lambda \widehat{D} \widehat{H})+\frac{1}{2 \lambda}\left(Q_{4}-\widetilde{D}_{x_{0} x_{0}}\left(2 Q_{3} Q_{1}+Q_{2}^{2}\right)+3 \widetilde{D}_{x_{0} x_{0}}^{2} Q_{2} Q_{1}^{2}-\widetilde{D}_{x_{0} x_{0}}^{3} Q_{1}^{4}\right) .
\end{aligned}
$$

Note that $S_{l}$ is a homogeneous polynomial of order $l$ in the $u_{x}$ 's and $H$ 's, assigning $u_{x}$ degree 1 and $H_{x y}$ degree 2 .

For $S_{1}$ and $S_{2}$ this gives in a first step

$$
\begin{aligned}
S_{1}[u, H] & =\frac{1}{2 \lambda} \sum_{x \neq x_{0}} u_{x}\left[1+\lambda \widehat{D}_{x x}-\widetilde{D}_{x_{0} x_{0}}^{-2} \widetilde{D}_{x_{0} x}^{2}\right] \\
S_{2}[u, H] & =\sum_{x, y \neq x_{0}} u_{x} u_{y}\left[-\frac{1}{4} \widehat{D}_{x y}^{2}+\frac{1}{2 \lambda} \frac{\widetilde{D}_{x_{0} x}}{\widetilde{D}_{x_{0} x_{0}}} \widetilde{D}_{x y} \frac{\widetilde{D}_{x_{0} y}}{\widetilde{D}_{x_{0} x_{0}}}-\frac{1}{2 \lambda} \widetilde{D}_{x_{0} x_{0}} \frac{\widetilde{D}_{x_{0} x}^{2}}{\widetilde{D}_{x_{0} x_{0}}^{2}} \frac{\widetilde{D}_{x_{0} y}^{2}}{\widetilde{D}_{x_{0} x_{0}}^{2}}\right] \\
& +\frac{1}{2} \sum_{x, y} H_{x y}\left[\lambda \widehat{D}_{x y}-\frac{\widetilde{D}_{x_{0} x}}{\widetilde{D}_{x_{0} x_{0}}} \frac{\widetilde{D}_{x_{0} y}}{\widetilde{D}_{x_{0} x_{0}}}\right]
\end{aligned}
$$


Using (3.13) one can now eliminate $\widetilde{D}_{x y}$ in favor of $D_{x y}:=\left(M^{-1}\right)_{x y}$. This produces an expression of the same form just with $D_{x y}$ replacing $\widetilde{D}_{x y}$. Next we eliminate $\widehat{D}_{x y}$ in favor of $D_{x y}$, using (3.10). Anticipating $D_{x_{0} x_{0}}=-1 / \lambda$ (which can be shown to hold independent of the gap equation) it follows that the saddle point equations $\delta S_{1} / \delta u_{x}=0$ are equivalent to

$$
-\lambda D_{x x}=1, \quad x \neq x_{0},
$$

which is the non-translation invariant precursor of the gap equation used in section 3.3. Inserting this into $S_{2}$ gives

$$
S_{2}[u, H]=-\frac{1}{4} \sum_{x, y \neq x_{0}} u_{x} u_{y}\left[D_{x y}^{2}-\lambda^{2} D_{x x_{0}}^{2} D_{y x_{0}}^{2}\right]+\frac{1}{2} \sum_{x, y} H_{x y} \lambda D_{x y} .
$$

So far translation invariance was not presupposed, i.e. we allowed for a nontrivial $x$ dependence in the extremal configurations $\omega_{x}, x \neq x_{0}$. We anticipate now from [4] that the only solution of (3.54) giving rise to a translation invariant $\left.\left\langle n_{x} \cdot n_{y}\right\rangle\right|_{N=\infty}=-\lambda D_{x y}$ is constant, i.e.

$$
\omega_{x}=\text { const }, \quad x \neq x_{0} .
$$

As seen in section 4.1 then $-\lambda D(x) \geq 1$ fixes the constant to be $\omega_{-}=\omega_{-}(\lambda, V)$, i.e. the unique solution of the translation invariant gap equation $-\lambda D(0)=1$ obeying $\omega_{-} \in\left(-4 \sin ^{2} \pi / L, 0\right)$. As before we write $D_{-}(x-y)$ for the translation invariant propagator with gap $\omega_{-}$. Further, in the appendix we prove that

$$
\left.S_{2}[u, H]\right|_{\omega_{x}=\omega_{-}} \geq 0
$$

This means that the saddle point is indeed a local minimum of the action (3.51). We conjecture that it is in fact a global minimum of the action (3.51) in the domain $\mathcal{D}(H)$.

We now return to the generating functional $W[H]$. Substituting (3.55) one arrives at

$$
\begin{aligned}
& \exp W[H]=\exp \left\{-\frac{1}{2} \sum_{x, y} H_{x y}\left[\lambda D_{-}(x-y)+1\right]\right\} \\
& \times \mathcal{N} \int \prod_{x \neq x_{0}} d u_{x} \exp \left\{+\frac{1}{4} \sum_{x, y \neq x_{0}} u_{x} u_{y} \widehat{D}_{-, 2}(x, y)\right\} \\
& \quad \times\left\{1+\frac{1}{N+1}\left(-S_{4}+\frac{1}{2} S_{3}^{2}\right)+O\left(\frac{1}{(N+1)^{2}}\right)\right\} .
\end{aligned}
$$

Here

$$
\widehat{D}_{-, 2}(x, y):=D_{-}(x-y)^{2}-\lambda^{2} D_{-}\left(x-x_{0}\right)^{2} D_{-}\left(y-x_{0}\right)^{2},
$$


and the terms occurring in the third line are generated by the expansion of

$$
\exp \left\{-\sum_{l \geq 3} \frac{1}{(N+1)^{\frac{l-2}{2}}} S_{l}[u, H]\right\}
$$

with the half-integer powers of $1 /(N+1)$ omitted. The conversion procedure exemplified for $S_{2}$ is easily seen to generalize to all $S_{l}, l \geq 3$. Indeed from $\left(\widetilde{A}^{-1}\right)_{x_{0} x_{0}}^{-1}=-\omega_{x_{0}}+$ $\left(A^{-1}\right)_{x_{0} x_{0}}^{-1}$ it follows that in an expansion

$$
\left(\widetilde{A}^{-1}\right)_{x_{0} x_{0}}^{-1}=\widetilde{D}_{x_{0} x_{0}}^{-1}-\frac{1}{\sqrt{N+1}} Q_{1}+\sum_{l \geq 2} \frac{1}{(N+1)^{l / 2}}\left[\left(\widetilde{A}^{-1}\right)_{x_{0} x_{0}}^{-1}\right]_{l}
$$

all coefficients $\left[\left(\widetilde{A}^{-1}\right)_{x_{0} x_{0}}^{-1}\right]_{l}, l \geq 2$, have the following properties: (i) eliminating $\widetilde{D}_{x y}$ in favor of $D_{x y}=\left(M^{-1}\right)_{x y}$ via (3.13), the parameter $\omega$ drops out. (ii) The result of the replacement is an expression of identically the same form, just with $D_{x y}$ occurring for each instance of $\widetilde{D}_{x y}$. (iii) In the result one can replace $D_{x y}$ by $D_{-}(x-y)$ and $\widehat{D}_{x y}$ by $D_{-}(x-y)+\lambda D_{-}\left(x-x_{0}\right) D_{-}\left(y-x_{0}\right)$.

The problem of computing $W[H]$ to order $1 /(N+1)^{p}$ thus reduces to the algebraic problem of computing the $S_{l}[u, H], l \leq 2 p+2$, with the indicated substitutions, and to performing Wick contractions with the free propagator

$$
\left\langle u_{x} u_{y}\right\rangle_{0}=-2 \widehat{\Delta}_{-}(x, y), \quad \sum_{z} \widehat{\Delta}_{-}(x, z) \widehat{D}_{-, 2}(z, y)=\delta_{x, y}, \quad x, y \neq x_{0}
$$

In fact one has

$$
\widehat{\Delta}_{-}(x, y)=\Delta_{-}(x-y), \quad \text { for } x, y \neq x_{0},
$$

where $\Delta_{-}$is the translation invariant free $u$-propagator defined by $\sum_{z} \Delta_{-}(x-z) D_{-}(z-$ $y)^{2}=\delta_{x y}$.

On the basis of the general results the $H$-moments computed with the algorithm described above must be translation invariant and be related to that of the compact model by the involution described. As a test of both the algorithm and the involution we now compute the two- and the four-point function to sub-leading order. To this end explicit expressions for $S_{3}$ and $S_{4}$ are needed.

It is convenient to parameterize them as follows

$$
\begin{aligned}
& S_{3}=S_{3}^{(0)}+S_{3}^{(1)}=\frac{1}{2}\left[\operatorname{Tr} p_{3}+\lambda Q_{3}^{(0)}+\lambda^{2} Q_{3}^{(1)}\right] \\
& S_{4}=S_{4}^{(0)}+S_{4}^{(1)}+S_{4}^{(2)}=\frac{1}{2}\left[\operatorname{Tr} p_{4}+\lambda Q_{4}^{(0)}+\lambda^{2} Q_{4}^{(1)}+\lambda^{3} Q_{4}^{(2)}\right] .
\end{aligned}
$$


Here $S_{l}^{(n)}, n=0,1,2$, are the pieces of order $n$ in the source $H$. The contribution to $S_{l}^{(n)}$ coming from $\left[\left(\widetilde{A}^{-1}\right)_{x_{0} x_{0}}^{-1}\right]_{l}$ in $(3.61)$ we denote by $Q_{l}^{(n)}$, for $n=0,1,2$, respectively. We need $Q_{3}^{(0)}, Q_{3}^{(1)}$ and $Q_{4}^{(1)}, Q_{4}^{(2)}$. They come out as

$$
\begin{aligned}
& Q_{3}^{(0)}=-\sum_{x, y, z \neq x_{0}} u_{x} u_{y} u_{z} D\left(x-x_{0}\right) D\left(z-x_{0}\right) \widehat{D}(x, y) \widehat{D}(y, z), \\
& Q_{3}^{(1)}=2 \sum_{x, y ; z \neq x_{0}} H_{x y} u_{z} D\left(x-x_{0}\right) D\left(z-x_{0}\right) \widehat{D}(y, z) \\
& Q_{4}^{(1)}=-\sum_{x, y ; z, w \neq x_{0}} H_{x y} u_{z} u_{w} D\left(z-x_{0}\right) \widehat{D}(w, x) \\
& \quad \times\left[2 D\left(y-x_{0}\right) \widehat{D}(z, w)+D\left(w-x_{0}\right) \widehat{D}(z, y)\right] \\
& Q_{4}^{(2)}=\sum_{x, y, z, w} H_{x y} H_{z w} D\left(x-x_{0}\right) D\left(w-x_{0}\right) \widehat{D}(y, z) .
\end{aligned}
$$

Here we omitted the subscript '-' on the propagators and we shall continue to do so for the remainder of this subsection.

For $S_{3}^{(0)}, S_{3}^{(1)}, S_{4}^{(1)}, S_{4}^{(2)}$ one obtains

$$
\begin{aligned}
& S_{3}^{(0)}=\frac{1}{6} \sum_{x, y, z \neq x_{0}} u_{x} u_{y} u_{z} \widehat{D}(x, y) \widehat{D}(y, z)\left[D(z-x)-2 \lambda D\left(x-x_{0}\right) D\left(z-x_{0}\right)\right], \\
& S_{3}^{(1)}=-\frac{\lambda}{2} \sum_{x, y ; z \neq x_{0}} H_{x y} u_{z} \widehat{D}(x, z)\left[D(z-y)-\lambda D\left(z-x_{0}\right) D\left(y-x_{0}\right)\right], \\
& S_{4}^{(1)}=\frac{\lambda}{4} \sum_{x, y ; z, w \neq x_{0}} H_{x y} u_{z} u_{w} \widehat{D}(x, w)\left\{\widehat{D}(z, w)\left[D(z-y)-3 \lambda D\left(z-x_{0}\right) D\left(y-x_{0}\right)\right]\right. \\
& \left.+\widehat{D}(z, y)\left[D(w-z)-\lambda D\left(w-x_{0}\right) D\left(z-x_{0}\right)\right]\right\}, \\
& S_{4}^{(2)}=-\frac{\lambda^{2}}{4} \sum_{x, y, z, w} H_{x y} H_{z w} \widehat{D}(y, z)\left[D(x-w)-\lambda D\left(x-x_{0}\right) D\left(w-x_{0}\right)\right] .
\end{aligned}
$$

The desired correlation functions can now be computed from

$$
\begin{aligned}
\left\langle-S_{4}^{(2)}+\frac{1}{2}\left[S_{3}^{(1)}\right]^{2}\right\rangle_{0} & =\frac{\lambda^{2}}{8} \sum_{x_{1}, y_{1}, x_{2}, y_{2}} H_{x_{1}, y_{1}} H_{x_{2}, y_{2}} W_{2}^{(0)}\left(x_{1}, y_{1} ; x_{2}, y_{2}\right), \\
\left\langle-S_{4}^{(1)}+S_{3}^{(0)} S_{3}^{(1)}\right\rangle_{0} & =\frac{\lambda}{2} \sum_{x, y} H_{x y} W_{1}^{(1)}(x, y) .
\end{aligned}
$$


For $W_{2}^{(1)}$ the $x_{0}$-dependent terms cancel out algebraically and one finds

$$
\begin{gathered}
W_{2}^{(0)}\left(x_{1}, y_{1} ; x_{2}, y_{2}\right)=D\left(x_{1}-x_{2}\right) D\left(y_{1}-y_{2}\right)+D\left(x_{1}-y_{2}\right) D\left(y_{1}-x_{2}\right) \\
-2 \sum_{z, w} D\left(x_{1}-z\right) D\left(y_{1}-z\right) \Delta(z-w) D\left(w-x_{2}\right) D\left(w-y_{2}\right) .
\end{gathered}
$$

The expansion of $\left\langle-S_{4}^{(1)}\right\rangle_{0}$ and $\left\langle S_{3}^{(0)} S_{3}^{(1)}\right\rangle_{0}$ generates a large number of $x_{0}$-dependent terms which simplify after using $\sum_{z} \Delta(x-z) D\left(z-x_{0}\right)^{2}=\delta_{x x_{0}}$. The results are

$$
\begin{aligned}
& \left\langle-S_{4}^{(1)}\right\rangle_{0}=\lambda \sum_{x y} H_{x y} \sum_{z, w} \Delta(z, w) D(z-w) D(x-w) D(y-z) \\
& -\lambda^{3} \sum_{x y} H_{x y} D\left(x-x_{0}\right) D\left(y-x_{0}\right) \sum_{z, w} \Delta(z, w) D(z-w) D\left(z-x_{0}\right) D\left(w-x_{0}\right) . \\
& \left\langle S_{3}^{(0)} S_{3}^{(1)}\right\rangle_{0}=-\lambda q \sum_{x y} H_{x y} \sum_{z} D(z-x) D(z-y) \\
& \quad+\lambda^{3} \sum_{x y} H_{x y} D\left(x-x_{0}\right) D\left(y-x_{0}\right) \sum_{z, w} \Delta(z, w) D(z-w) D\left(z-x_{0}\right) D\left(w-x_{0}\right) .
\end{aligned}
$$

Here

$$
q:=\sum_{z} \Delta(z) \sum_{u, v} D(z-u) D(z-v) \Delta(u-v) D(u-v)
$$

Finally

$$
\begin{aligned}
& W_{1}^{(1)}(x, y)=-2 q \sum_{w} D(x-w) D(y-w) \\
& \quad+2 \sum_{z, w} D(x-z) D(y-w) \Delta(z-w) D(z-w),
\end{aligned}
$$

which obeys $W_{1}^{(1)}(x, x)=0$. Recall that $D(x)$ here is short for $D_{-}(x)=\left.D(x)\right|_{\omega=\omega_{-}}$. The results (3.71) and (3.68) are manifestly translation invariant and related to those of the compact model (see $[18,9]$ ) by the involution described.

\section{Conclusions}

On the level of asymptotic expansions interesting and useful correspondences exist between compact and noncompact nonlinear sigma-models. Here we established two such 
correspondences: in perturbation theory, where simply flipping the sign of $\beta$ allows to move between the compact and noncompact models, and in the large $N$ expansion, where one has to make a detour through the finite volume in order to establish a correspondence that involves not just flipping the sign of $\lambda$ but also picking the physically appropriate solution of the gap equation.

Taking the sign flip prescription beyond perturbation theory leads from the compact model not to the noncompact one, but rather to another compact model, the antiferromagnet.

In the literature [19] a sign flip rule for the large $N$ coefficients in a continuum formulation has been proposed. By section 3.4 the rule is incorrect. In [2] the much weaker claim has been made that there exists a scheme in which the coefficients of the large $N$ beta function in the compact and in the noncompact models are related by the sign flip $\lambda \rightarrow-\lambda$. This statement seems to be in agreement with our findings because it is based on re-organizing the perturbation expansion into powers of $1 / N$, but it remains an open question whether the analytic continuation from positive to negative $\lambda$ employed in [2] to go from the compact to the noncompact model is justified in the continuum.

Acknowledgements: We wish to thank A. Duncan and M. Lüscher for discussions and correspondence. 


\section{Appendix A: Positivity of $S_{2}$}

Here we show that

$$
\left.S_{2}[u, H]\right|_{\omega_{x}=\omega_{-}} \geq 0
$$

which guarantees that the saddle point on which the large $N$ expansion is based is indeed a local minimum of the action (3.51).

We begin by noting that the source-dependent term separately obeys

$$
\frac{1}{2} \sum_{x, y} H_{x y} \lambda D(x-y) \geq 0
$$

for all sources $H_{x y} \leq 0$, as $-\lambda D(x-y) \geq 1$. It therefore suffices to consider $S_{2}[u, 0]$. Next we introduce

$$
\Pi(p):=\sum_{x} e^{i p x} D(x)^{2}=\frac{1}{V} \sum_{k} \frac{1}{\left(E_{k}+\omega\right)\left(E_{k-p}+\omega\right)},
$$

and claim that in terms of it a sufficient condition for $S_{2}[u, 0] \geq 0$ is

$$
\Pi(p)<0, \quad p \neq 0 .
$$

To see this we prepare

$$
\Pi(0) \geq \frac{V}{\lambda^{2}}
$$

using once more $1 \leq-\lambda D(x)$. Then we rewrite $S_{2}[u, 0]$ in Fourier space

$$
\begin{aligned}
S_{2}[u, 0] & =\frac{1}{4 V^{2}} \sum_{p, q} u(p)^{*} \mathcal{S}_{p q} u(q), \\
\mathcal{S}_{00} & =-\Pi(0)\left[V-\lambda^{2} \Pi(0)\right], \\
\mathcal{S}_{0 p} & =\lambda^{2} v_{0} v_{p}, \quad v_{p}:=\Pi(p) e^{-i p x_{0}}, \\
\mathcal{S}_{p q} & =-\delta_{p q} V \Pi(p)+\lambda^{2} v_{p}^{*} v_{q}, \quad p, q \neq 0 .
\end{aligned}
$$

Subject to (A.4) and (A.5) the matrix $\mathcal{S}$ has the form of a positive rank one perturbation of a positive diagonal matrix, which therefore must be itself positive.

It remains to show (A.4). We rewrite $\Pi(p)$ in the form

$$
\begin{aligned}
\Pi(p) & =-\frac{1}{E_{p}+2 \omega}\left[\frac{2}{\lambda}+J(p)\right], \quad p \neq 0, \\
J(p) & =\frac{1}{V} \sum_{k} \frac{E_{k}+E_{p-k}-E_{p}}{\left(E_{k}+\omega\right)\left(E_{p-k}+\omega\right)},
\end{aligned}
$$


using the gap equation. Here $J(p)$ manifestly has a finite thermodynamic limit. Further we claim

$$
J(p) \geq 0 \text { for all } p,
$$

which implies (A.4).

The proof of (A.8) is based on a reorganization of the $d$-fold sum in (A.7) such that each term is positive on account of the Lemma below. We begin by noting that $J(p)$ is completely symmetric in all arguments $p=\left(p_{1}, \ldots, p_{d}\right)$, and that

$$
J\left(\left(2 \pi-p_{1}, \tilde{p}_{1}\right)\right)=J(p), \quad \tilde{p}_{\mu}:=\left(p_{1}, \ldots, p_{\mu-1}, p_{\mu+1}, \ldots, p_{d}\right) .
$$

Since $J(0)>0$ trivially, it suffices to restrict attention to momenta $p \neq 0$ with $0 \leq p_{1} \leq$ $\ldots \leq p_{d} \leq \pi$. Further, from

$$
E_{p-k}+E_{k}-E_{p}=8 \sum_{\mu} \cos \frac{p_{\mu}}{2} \sin \frac{k_{\mu}}{2} \sin \frac{(k-p)_{\mu}}{2}
$$

one sees that for $L$ even

$$
J((\pi, \ldots, \pi))=0, \quad J((0, \pi, \ldots, \pi))>0 .
$$

Next we insert (A.10) into (A.7) to obtain

$$
\begin{aligned}
J(p) & =\frac{1}{4} \sum_{\mu} \frac{1}{L^{d-1}} \sum_{k_{\nu}, \nu \neq \mu} S\left(\tilde{k}_{\mu} \mid p\right), \\
S\left(\tilde{k}_{\mu} \mid p\right) & =\frac{1}{L} \sum_{k_{\mu}} \frac{2 \cos \frac{p_{\mu}}{2} \sin \frac{k_{\mu}}{2} \sin \frac{k_{\mu}-p_{\mu}}{2}}{\left[\sin ^{2} \frac{k_{\mu}}{2}-\sin ^{2} \frac{\alpha}{2}+X_{\mu}\right]\left[\sin ^{2} \frac{k_{\mu}-p_{\mu}}{2}-\sin ^{2} \frac{\alpha}{2}+Y_{\mu}\right]},
\end{aligned}
$$

where we set

$$
X_{\mu}:=\sum_{\nu \neq \mu} \sin ^{2} \frac{k_{\nu}}{2}, \quad Y_{\mu}:=\sum_{\nu \neq \mu} \sin ^{2} \frac{k_{\nu}-p_{\nu}}{2}, \quad 2 \sin \frac{\alpha}{2}:=\sqrt{-\omega}
$$

The result (A.8) now follows from the

Lemma: For all $L \geq 2$ and $q \in[0, \pi], q \in \frac{2 \pi}{L} \mathbb{Z}$,

$$
S(q, \alpha, x, y):=\frac{1}{L} \sum_{n=0}^{L-1} \frac{2 \cos \frac{q}{2} \sin \frac{\pi n}{L} \sin \left(\frac{\pi n}{L}-\frac{q}{2}\right)}{\left[\sin ^{2} \frac{\pi n}{L}-\sin ^{2} \frac{\alpha}{2}+x\right]\left[\sin ^{2}\left(\frac{\pi n}{L}-\frac{q}{2}\right)-\sin ^{2} \frac{\alpha}{2}+y\right]} \geq 0
$$

for parameters $x, y \geq 0$ and $0<\alpha<\pi / L$. 
In the application to (A.12) only the bound on $\alpha$ needs to be verified. From (3.28) we have however

$$
\sin \frac{\alpha}{2}<\sqrt{\frac{4}{2 d+1}} \sin \frac{\pi}{2 L} .
$$

Proof of the Lemma. Trivially $S(0, \alpha, x, y)>0$ and $S(\pi, \alpha, x, y)=0$ for $L$ even. It thus suffices to consider $q \in(0, \pi)$. We make use of the following summation formulas:

$$
\frac{1}{L} \sum_{n=0}^{L-1} \frac{1}{\sin ^{2} \frac{\pi n}{2}+\operatorname{sh}^{2} \frac{a}{2}}=\frac{2 \operatorname{coth} \frac{L a}{2}}{\operatorname{sh} a}, \quad a \neq 0
$$

and for $q \in \frac{2 \pi}{L} \mathbb{Z}$ and $a \neq b \neq 0$ :

$$
\begin{aligned}
& \frac{1}{L} \sum_{n=0}^{L-1} \frac{1}{\left[\sin ^{2} \frac{\pi n}{L}+\operatorname{sh}^{2} \frac{a}{2}\right]\left[\sin ^{2}\left(\frac{\pi n}{L}-\frac{q}{2}\right)+\operatorname{sh}^{2} \frac{b}{2}\right]} \\
& \quad=\frac{2}{\operatorname{sh} \frac{L a}{2} \operatorname{sh} \frac{L b}{2} \operatorname{sh} a \operatorname{sh} b}\left[\frac{\operatorname{sh} \frac{L}{2}(a+b) \operatorname{sh}(a+b)}{\operatorname{ch}(a+b)-\cos q}+\frac{\operatorname{sh} \frac{L}{2}(a-b) \operatorname{sh}(a-b)}{\operatorname{ch}(a-b)-\cos q}\right] .
\end{aligned}
$$

For $a=b \neq 0$ the result of the summation is obtained from the rhs of (A.17) by omitting the second term in square brackets and specializing the remainder to $a=b$ (i.e. no pole occurs for $a=b$ and $q=0$ ). Both (A.16) and (A.17) can be obtained from

$$
\frac{1}{L} \sum_{n=0}^{L-1} \frac{1}{e^{\frac{2 \pi i n}{L}}-a}=\frac{a^{L-1}}{1-a^{L}}, \quad a^{L} \neq 1
$$

In the application to $S$ we set $\operatorname{sh}^{2} \frac{a}{2}=x-\sin ^{2} \frac{\alpha}{2}, \operatorname{sh}^{2} \frac{b}{2}=y-\sin ^{2} \frac{\alpha}{2}$, and pick the roots $a, b>0$ for $x, y>\sin ^{2} \frac{\alpha}{2}$. In a first step one obtains (also using the $1 \mathrm{~d}$ version of (A.10) backwards)

$$
\begin{aligned}
S= & \frac{2 \operatorname{coth} \frac{L a}{2}}{\operatorname{sh} a}+\frac{2 \operatorname{coth} \frac{L b}{2}}{\operatorname{sh} b}-\frac{2\left(\operatorname{sh}^{2} \frac{a}{2}+\operatorname{sh}^{2} \frac{b}{2}+\sin ^{2} \frac{q}{2}\right)}{\operatorname{sh} \frac{L a}{2} \operatorname{sh} \frac{L b}{2} \operatorname{sh} a \operatorname{sh} b} \\
& \times\left[\frac{\operatorname{sh} \frac{L}{2}(a+b) \operatorname{sh}(a+b)}{\operatorname{ch}(a+b)-\cos q}+\frac{\operatorname{sh} \frac{L}{2}(a-b) \operatorname{sh}(a-b)}{\operatorname{ch}(a-b)-\cos q}\right] .
\end{aligned}
$$

This can be rewritten in the form

$$
S=\frac{\cos ^{2} \frac{q}{2}}{\operatorname{ch} \frac{a}{2} \operatorname{ch} \frac{b}{2} \operatorname{sh} \frac{L a}{2} \operatorname{sh} \frac{L b}{2}}\left[\frac{\operatorname{sh} \frac{L}{2}(a+b) \operatorname{sh} \frac{a+b}{2}}{\operatorname{sh}^{2} \frac{a+b}{2}+\sin ^{2} \frac{q}{2}}-\frac{\operatorname{sh} \frac{L}{2}(a-b) \operatorname{sh} \frac{a-b}{2}}{\operatorname{sh}^{2} \frac{a-b}{2}+\sin ^{2} \frac{q}{2}}\right] .
$$

Since the function

$$
t(z):=\frac{\operatorname{sh} \frac{L z}{2} \operatorname{sh} \frac{z}{2}}{\operatorname{sh}^{2} \frac{z}{2}+\sin ^{2} \frac{q}{2}}
$$


is positive and monotonically increasing on $\mathbb{R}_{+}$it follows that for $a, b>0$ and $q \in(0, \pi)$ the rhs of (A.20) is nonnegative.

Next consider the case where $0<\beta:=i b<\pi / L$ and $a>0$. The expression (A.20) can in this case be written as

$$
\begin{aligned}
S & =\frac{4 \cos ^{2} \frac{q}{2}}{\operatorname{ch} \frac{a}{2} \cos \frac{\beta}{2}} \frac{\operatorname{coth} \frac{L a}{2} s_{1}-\cot \frac{L \beta}{2} s_{2}}{(\operatorname{ch} a \cos \beta-\cos q)^{2}+\operatorname{sh}^{2} a \sin ^{2} \beta}, \\
s_{1} & =\operatorname{sh} \frac{a}{2} \cos \frac{\beta}{2}[\operatorname{ch} a-\cos \beta+1-\cos q], \\
s_{2} & =\operatorname{ch} \frac{a}{2} \sin \frac{\beta}{2}[\operatorname{ch} a-\cos \beta-1+\cos q] .
\end{aligned}
$$

Further

$$
\begin{aligned}
& \operatorname{coth} \frac{L a}{2} s_{1}-\cot \frac{L \beta}{2} s_{2}=(\operatorname{ch} a-\cos \beta) \operatorname{ch} \frac{a}{2} \cos \frac{\beta}{2}\left[\operatorname{coth} \frac{L a}{2} \operatorname{th} \frac{a}{2}-\cot \frac{L \beta}{2} \tan \frac{\beta}{2}\right] \\
& +(1-\cos q)\left[\operatorname{coth} \frac{L a}{2} \operatorname{sh} \frac{a}{2} \cos \frac{\beta}{2}+\cot \frac{L \beta}{2} \sin \frac{\beta}{2} \operatorname{ch} \frac{a}{2}\right] .
\end{aligned}
$$

The second term in (A.23) is manifestly positive. For the first term we note that $\beta \mapsto$ $\cot \frac{L \beta}{2} \tan \frac{\beta}{2}$ maps $[0, \pi / L]$ bijectively onto $[1 / L, 0]$, while $a \mapsto \operatorname{coth} \frac{L a}{2} \operatorname{th} \frac{a}{2}$ maps $[0, \infty)$ bijectively onto $[1 / L, \infty)$. Hence

$$
\operatorname{coth} \frac{L a}{2} \operatorname{th} \frac{a}{2}-\cot \frac{L \beta}{2} \tan \frac{\beta}{2} \geq 0
$$

which shows that $S$ in (A.22) is nonnegative.

It remains to consider the case $0<\beta:=i b<\pi / L$, and $0<\alpha:=i a<\pi / L$, (which may differ from the $\alpha$ used in the formulation of the Lemma). Specialization of (A.20) gives

$$
S=\frac{\cos ^{2} \frac{q}{2}}{\cos \frac{\alpha}{2} \cos \frac{\beta}{2} \sin \frac{L \alpha}{2} \sin \frac{L \beta}{2}}\left[\frac{\sin \frac{L}{2}(\alpha+\beta) \sin \frac{\alpha+\beta}{2}}{\sin ^{2} \frac{q}{2}-\sin ^{2} \frac{\alpha+\beta}{2}}-\frac{\sin \frac{L}{2}(\alpha-\beta) \sin \frac{\alpha-\beta}{2}}{\sin ^{2} \frac{q}{2}-\sin ^{2} \frac{\alpha-\beta}{2}}\right] .
$$

Since $x \mapsto-t(i x)$ (with $t$ from (A.21)) is again positive and strictly increasing for $x \in[0, \pi / L]$ and $q \in(0, \pi)$, it follows that the rhs of (A.25) is non-negative for the range of $\alpha, \beta$ considered. This concludes the proof of the Lemma. 


\section{References}

[1] A. Duncan, M. Niedermaier, E. Seiler, Vacuum orbit and spontaneous symmetry breaking in hyperbolic sigma-models, Nucl. Phys. B720 (2005) 235.

[2] J. J. Friess and S. S. Gubser, Non-linear sigma models with anti-de Sitter target spaces, Nucl. Phys. B750[FS] (2006) 111.

[3] T. Spencer, M.R. Zirnbauer, Spontaneous symmetry breaking of a hyperbolic sigma model in three dimensions, Commun. Math. Phys. 252 (2004) 167-187.

[4] M. Niedermaier and E. Seiler, in preparation.

[5] A. Houghton, A. Jevicki, R. Kenway, A. Pruisken, Noncompact sigma-models and the existence of a mobility edge in disordered electron systems near two dimensions, Phys. Rev. Lett 45 (1980) 394.

[6] S. Hikami, Three loop beta function of nonlinear sigma-models on symmetric spaces, Phys. Lett. B98 (1981) 208.

[7] S. Elitzur, The applicability of perturbation expansion to two-dimensional Goldstone systems, Nucl.Phys. B212 (1983) 501.

[8] F. David, Cancellation of infrared divergences in the 2D nonlinear sigma-model, Commun. Math. Phys. 81 (1981) 149.

[9] A. Duncan, M. Niedermaier, and P. Weisz, Noncompact sigma-models - Large $N$ expansion and thermodynamic limit, in preparation.

[10] S. Kobayashi and K. Nomizu, Foundations of Differential Geometry, Vol. II., Interscience, New York 1969.

[11] S. Helgason, Differential Geometry and Symmetric Spaces, academic Press, New York 1962.

[12] E. T. Copson, Asymptotic Expansions, Cambridge University Press, Cambridge 1965.

[13] J. D. Murray, Asymptotic Analysis, Oxford University Press, Oxford 1974.

[14] H. Mukaida and Y. Shimada, Convex effective potential of $O(N)$ symmetric $\phi^{4}$ theory for large $N$, Nucl. Phys. B479 [FS] (1996) 663.

[15] A. Duncan, E. Seiler and P. Weisz, unpublished notes. 2005.

[16] A. J. Kupiainen, On the 1/n expansion, Commun. Math. Phys. 73 (1980) 273.

[17] M. Lüscher, Schwinger-Dyson equations in a finite volume, unpublished notes $1980 / 81$. 
[18] G. Cristofano, R. Musto, F. Nicodemi, R. Pettorino, and F. Pezzella, $1 / N$ contribution to physical quantities in the lattice $\mathrm{O}(N)$ sigma-model, Nucl. Phys. 257 [FS14] (1985) 505 .

[19] S.A. Brunini, M. Gomes, A.J. da Silva, Remarks on non-compact sigma-models, Phys. Rev. D38 (1988) 706. 